\title{
Harmonic Bergman spaces, the Poisson equation and the dual of Hardy-type spaces on certain noncompact manifolds
}

\author{
Giancarlo Mauceri, Stefano Meda And Maria VAllarino
}

\begin{abstract}
In this paper we consider a complete connected noncompact Riemannian manifold $M$ with bounded geometry and spectral gap. We realize the dual space $Y^{k}(M)$ of the Hardy-type space $X^{k}(M)$, introduced in a previous paper of the authors, as the class of all locally square integrable functions satisfying suitable BMO-like conditions, where the role of the constants is played by the space of global $k$-quasi-harmonic functions. Furthermore we prove that $Y^{k}(M)$ is also the dual of the space $X_{\text {fin }}^{k}(M)$ of finite linear combination of $X^{k}$-atoms. As a consequence, if $Z$ is a Banach space and $T$ is a $Z$-valued linear operator defined on $X_{\text {fin }}^{k}(M)$, then $T$ extends to a bounded operator from $X^{k}(M)$ to $Z$ if and only if it is uniformly bounded on $X^{k}$-atoms. To obtain these results we prove the global solvability of the generalized Poisson equation $\mathscr{L}^{k} u=f$ with $f \in L_{\mathrm{loc}}^{2}(M)$ and we study some properties of generalized Bergman spaces of harmonic functions on geodesic balls.
\end{abstract}

Mathematics Subject Classification (2010): 30H10 (primary); 42B20, 42B35, $58 \mathrm{C} 99$ (secondary).

\section{Introduction}

A seminal result of $\mathrm{C}$. Fefferman [10, Theorem 2] identifies the Banach dual of the Hardy space $H^{1}\left(\mathbb{R}^{n}\right)$ as $\mathrm{BMO}\left(\mathbb{R}^{n}\right) / \mathbb{C}$, the space of all functions of bounded mean oscillation modulo constants. A function $g$ in $\operatorname{BMO}\left(\mathbb{R}^{n}\right)$ possesses the nice property of being "well approximated" on each ball by constants, to wit

$$
\sup _{B} \inf _{c \in \mathbb{C}} \frac{1}{|B|} \int_{B}|g(x)-c|^{2} \mathrm{~d} x<\infty .
$$

Work partially supported by PRIN 2010 "Real and complex manifolds: geometry, topology and harmonic analysis". The authors are members of the Gruppo Nazionale per l'Analisi Matematica, la Probabilità e le loro Applicazioni (GNAMPA) of the Istituto Nazionale di Alta Matematica (INdAM).

Received January 19, 2013; accepted in revised form November 25, 2013. 
The continuous linear functionals on $H^{1}\left(\mathbb{R}^{n}\right)$ are precisely those which, restricted to finite linear combinations $f$ of $H^{1}$-atoms, are of the form

$$
\lambda_{g}(f):=\int_{\mathbb{R}^{n}} f(x) g(x) \mathrm{d} x
$$

for some function $g$ in $\operatorname{BMO}\left(\mathbb{R}^{n}\right)$.

Recently several authors have investigated Hardy spaces on noncompact doubling Riemannian manifolds $[2,14,22]$. On nondoubling manifolds, versions of the so called local Hardy spaces, introduced by D. Goldberg in the context of $\mathbb{R}^{n}[11]$, have been investigated in $[7,26]$. These local Hardy spaces are well adapted to obtain endpoint estimates for singular integrals whose kernels have only local singularities, such as, for instance, pseudodifferential operators. However, it is known that singular integral operators whose kernel is also singular at infinity, such as Riesz transforms or imaginary powers of the Laplacian, do not map the local Hardy spaces in $L^{1}(M)$. To overcome this problem, in [16,17] the authors introduced and studied the properties of a family of global Hardy-type spaces on a class of nondoubling manifolds that includes all symmetric spaces of the noncompact type.

In this paper we aim at proving a version of Fefferman's result for this new class of spaces. A striking difference between the aforementioned classical result and our version thereof is that the role played by constants in the former will be played in the latter by quasi-harmonic functions, i.e., solutions to the (generalised) Poisson equation $\mathscr{L}^{k} u=c$ for some positive integer $k$ and constant $c$. Here $\mathscr{L}$ denotes minus the Laplace-Beltrami operator on $M$.

We elaborate on this. In $[16,17]$ we defined a strictly decreasing sequence $X^{1}(M), X^{2}(M), X^{3}(M), \ldots$ of subspaces of $L^{1}(M)$, where $M$ is a complete connected noncompact Riemannian manifold with Ricci curvature bounded from below, positive injectivity radius and spectral gap (see also [27] for an interesting variant of the spaces $X^{k}(M)$ ). Note that these manifolds are of exponential volume growth, hence their Riemannian measure $\mu$ is nondoubling. Important examples of manifolds with these properties are nonamenable connected unimodular Lie groups equipped with a left invariant Riemannian distance, and symmetric spaces of the noncompact type with the Killing metric. The spaces $X^{k}(M)$ share with the classical Hardy space $H^{1}\left(\mathbb{R}^{n}\right)$ the following properties (see $\left.[16,17]\right)$ :

(i) if $p$ is in $(1,2)$, then the Lebesgue space $L^{p}(M)$ is an interpolation space between $X^{k}(M)$ and $L^{2}(M)$;

(ii) some interesting operators, such as the Riesz transforms associated to $\mathscr{L}$ and the purely imaginary powers of $\mathscr{L}$, are bounded from $X^{k}(M)$ to $L^{1}(M)$ for $k$ large enough (improvements thereof will appear in [18]);

(iii) the space $X^{1}(M)$ admits an atomic decomposition in terms of atoms, which are defined much as in the classical case, but are supported in balls of radius at most one and satisfy an appropriate infinite dimensional cancellation condition. Under the additional assumption that some (depending on $k$ ) covariant derivatives of the Ricci tensor are bounded, the same holds for $X^{k}(M)$ with $k \geq 2$. 
For this reason we call the spaces $X^{k}(M)$ generalised Hardy spaces. They play for harmonic analysis on $M$ a role similar to that played by $H^{1}\left(\mathbb{R}^{n}\right)$ on $\mathbb{R}^{n}[24]$ and, more generally, by the Coifman-Weiss Hardy space [8] on spaces of homogeneous type.

In order to describe the cancellation condition alluded to in (iii) above we define, for each geodesic ball $B$, the class $q_{k}^{2}(\bar{B})$ of all $k$-quasi-harmonic functions on $\bar{B}$ as the class of functions that are restrictions to $\bar{B}$ of functions $v$ such that

$$
\mathscr{L}^{k} v=\text { const }
$$

in some open neighbourhood of $\bar{B}$.

Atoms in $X^{k}(M)$ are then $L^{2}(M)$ functions $A$ with support contained in a ball $B$ of radius at most one such that

(i) $\int A v \mathrm{~d} \mu=0 \quad \forall v \in q_{k}^{2}(\bar{B})$;

(ii) $\|A\|_{2} \leq \mu(B)^{-1 / 2}$.

The space $X^{k}(M)$ is the space of all (possibly infinite) linear combinations of $X^{k}$ atoms with $\ell^{1}$ coefficients, endowed with the standard "atomic norm". If we consider atoms with support contained in balls of radius at most $s>0$, instead of atoms with support in balls of radius at most 1 , we obtain the same space of functions, and the two corresponding "atomic norms" are equivalent [17]. In view of this observation, we may choose the "scale parameter" $s$ equal to $s_{0}:=(1 / 2) \operatorname{Inj}(M)$. This will simplify some of the arguments below, and avoid many annoying technicalities. We shall call atoms supported in balls od radius at most $s_{0}$ admissible.

An important point that we overlooked in [17], and that we shall discuss in Section 4 , is that the cancellation condition for $X^{k}$-atoms may be equivalently formulated, at least for atoms $A$ with support in small balls, by requiring that $A$ is orthogonal to $q_{k}(M)$, the space of all global $k$-quasi- harmonic functions, i.e. the space of all solutions to the (generalised) Poisson equation

$$
\mathscr{L}^{k} u=c
$$

where $c$ is an arbitrary constant. In Section 2 we shall prove that the generalised Poisson equation has global solutions and that if $r_{B}$ is small enough, then functions in $q_{k}^{2}(\bar{B})$ may be approximated in the $L^{2}(B)$ norm to any degree of precision by global $k$-quasi-harmonic functions. This suggests to define the generalised BMO space $\operatorname{GBMO}^{k}(M)$ as the space of all locally square integrable functions $G$ on $M$ such that

$$
\|G\|_{\mathrm{GBMO}^{k}}(M):=\sup _{B} \inf _{V \in q_{k}(M)}\left(\frac{1}{\mu(B)} \int_{B}|G-V|^{2} \mathrm{~d} \mu\right)^{1 / 2}<\infty,
$$

where the supremum is taken with respect to all balls of radius at most $s_{0}$. Note that this "norm" annihilates all global $k$-quasi-harmonic functions, and defines a genuine 
norm on the quotient space $\mathrm{GBMO}^{k}(M) / q_{k}(M)$. Loosely speaking, functions in $\mathrm{GBMO}^{k}(M)$ may be "well approximated" on balls of radius at most $s_{0}$ by global $k$-quasi-harmonic functions. Our main result, Theorem 5.8, states that the Banach dual of $X^{k}(M)$ is isomorphic to $\mathrm{GBMO}^{k}(M) / q_{k}(M)$. Specifically, the continuous linear functionals on $X^{k}(M)$ are precisely those, which, restricted to finite linear combinations $F$ of $X^{k}$ - atoms, are of the form

$$
\lambda_{G}(F):=\int_{M} F G \mathrm{~d} \mu
$$

for some function $G$ in $\mathrm{GBMO}^{k}(M)$. Note the analogy between the classical case (1.1), (1.2), and our setting (1.3), (1.4). It is an interesting problem to determine explicitly the function $G$ that corresponds to a given functional $\lambda$. We solve this problem in Section 5. It may be worth observing that one of the steps in the proof is showing that the solutions of the generalised Poisson equation

$$
\mathscr{L}^{k} u=g
$$

with datum $g$ in $\operatorname{BMO}(M)$ are in $\operatorname{GBMO}^{k}(M)$, with control of the norms. As a consequence of our analysis, we prove in Section 6 that the $X^{k}(M)$-norm and the norm

$$
\inf \left\{\sum_{j}\left|c_{j}\right|: F=\sum_{j} c_{j} A_{j}\right\},
$$

where the infimum is taken over all representations of $F$ as a finite sum of admissible $X^{k}$-atoms, are equivalent on the space $X_{\text {fin }}^{k}(M)$ of finite linear combination of $X^{k}$-atoms. This implies that if $Z$ is a Banach space and $\mathscr{T}$ is a $Z$-valued linear operator on finite combinations of $X^{k}$-atoms that is uniformly bounded on admissible $X^{k}$-atoms, then it extends to a bounded linear operator from $X^{k}(M)$ to $Z$. Thus, the atomic decomposition is really useful to test the boundedness of linear operators defined on finite linear combinations of $X^{k}$ - atoms. See, on this delicate point, $[15,20,21]$ and the references therein. This result has already been implicitly used in $[16,17]$, where, in order to show that certain singular integral operators are bounded from $X^{k}(M)$ to $L^{1}(M)$, we simply checked that they are uniformly bounded on $X^{k}$-atoms.

Further applications of the theory developed in this paper to the boundedness of spectral multipliers of $\mathscr{L}$ and Riesz transforms will appear in [19].

We briefly outline the content of this paper. Section 2 is devoted to the study of the solvability of the generalised Poisson equation. In Section 3 we introduce various classes of $k$-quasi-harmonic functions and study their mutual relations. In Section 4 , after stating the basic geometric assumptions on the manifold $M$ and their analytic consequences, we recall the definition of the spaces $H^{1}(M), X^{k}(M)$, $Y^{k}(M)$ and their main properties. Our main result is proved in Section 5. Finally, in Section 6 we shall prove that the dual of $X_{\text {fin }}^{k}(M)$ is isomorphic to that of $X^{k}(M)$, and draw some consequences concerning the extendability of Banach-valued linear operators uniformly bounded on $X^{k}$-atoms. 
We shall use the "variable constant convention", and denote by $C$, possibly with sub- or superscripts, a constant that may vary from place to place and may depend on any factor quantified (implicitly or explicitly) before its occurrence, but not on factors quantified afterwards. Throughout the paper $\mathbf{1}_{E}$ will denote the indicator function of the set $E$.

\section{Solvability of the Poisson equation}

In this section $M$ will denote a connected complete $n$-dimensional Riemannian manifold of infinite volume with Riemannian measure $\mu$. We assume that the bottom $b$ of the $L^{2}(M)$ spectrum of $\mathscr{L}$ is strictly positive and so $\mathscr{L}^{-1}$ is bounded on $L^{2}(M)$. The aim of this section is to investigate the solvability of the (generalised) Poisson equation

$$
\mathscr{L}^{k} u=g \text {, }
$$

where $g$ is a datum in $L_{\text {loc }}^{2}(M)$ and $k$ a positive integer. Clearly, if $U$ is a distributional solution of this equation, any other solution is of the form $U+H$, where $H$ solves the generalised Laplace equation $\mathscr{L}^{k} H=0$, i.e. $H$ is a global $k$-harmonic function on $M$, according to the terminology that we shall introduce in Definition 3.1 .

The proof of the solvability hinges on the following approximation result of $k$-harmonic functions on certain compact subsets of $M$ by global $k$-harmonic functions on $M$. The proof for $k=1$ can be found in [3, Theorem 3.10]; the case $k>1$ is a straightforward adaptation of the argument given there. We recall that if $K$ is a closed subset of $M$, then a hole of $K$ is any component of $M \backslash K$ which is bounded.

Lemma 2.1 (Walsch-Pfluger-Lax-Malgrange). Let $K$ be a compact subset of $M$ without holes and $k$ be a positive integer. If $v$ is a solution of the equation $\mathscr{L}^{k} v=0$ in a neighbourhood of $K$ and $\varepsilon>0$ then there is a function $u$ such that $\mathscr{L}^{k} u=0$ in $M$ and $\sup _{K}|v-u|<\varepsilon$.

Theorem 2.2. Suppose that $M$ is a complete, noncompact, Riemannian manifold with spectral gap and that $k$ is a positive integer. Then for every $f$ in $L_{\mathrm{loc}}^{2}(M)$ there exists $u$ in $L_{\text {loc }}^{2}(M)$ such that $\mathscr{L}^{k} u=f$ in the sense of distributions.

Proof. First we consider the case $k=1$. Fix a reference point $o$ in $M$ and denote by $B_{R}$ the open ball of radius $R$ and centre $o$, and by $\widehat{B}_{R}$ the union of $B_{R}$ with the bounded connected components of $M \backslash B_{R}$. Then $\widehat{B}_{R}$ has no holes and $M$ is the union of the increasing sequence of bounded open sets $\left\{\widehat{B}_{R}\right\}$.

Fix $\varepsilon>0$ small. Set $v_{1}=\mathscr{L}^{-1}\left(f \mathbf{1}_{\widehat{B}_{1+\varepsilon}}\right)$. This makes sense, because $\mathscr{L}^{-1}$ is bounded on $L^{2}(M)$. Then $v_{1}$ is in $L^{2}(M)$ and solves the equation $\mathscr{L} v_{1}=f$ in $\widehat{B}_{1+\varepsilon}$. If $w \in L^{2}(M)$ is a solution of $\mathscr{L} w=f$ in $\widehat{B}_{2+\varepsilon}$ (for instance $w=$ $\left.\mathscr{L}^{-1}\left(f \mathbf{1}_{\widehat{B}_{2+\varepsilon}}\right)\right)$, then $\mathscr{L}\left(v_{1}-w\right)=0$ in $\widehat{B}_{1+\varepsilon}$. Hence, by Lemma 2.1 , there exists $h$ such that $\mathscr{L} h=0$ in $M$ and $\sup _{\widehat{B}_{1}}\left|v_{1}-w-h\right|<1 / 2$. Thus, setting $v_{2}=w+h$, 
one has $\mathscr{L} v_{2}=f$ in $\widehat{B}_{2+\varepsilon}$ and $\sup _{\widehat{B}_{1}}\left|v_{1}-v_{2}\right|<1 / 2$. Iterating this argument, one constructs a sequence of functions $v_{j}$ in $L^{2}(M)$ such that $\mathscr{L} v_{j}=f$ in $\widehat{B}_{j+\varepsilon}$ and $\sup _{\widehat{B}_{j}}\left|v_{j}-v_{j+1}\right|<2^{-j}$. Thus $v_{j}$ converges in $L_{\text {loc }}^{2}(M)$, whence in the sense of distributions, to a limit $u$, which satisfies $\mathscr{L} u=f$ in $M$.

The case $k>1$ can be reduced to $k=1$, by observing that the equation $\mathscr{L}^{k} u=f$ is equivalent to the system of $k$ equations $\mathscr{L} u_{\ell}=u_{\ell-1}, \ell=1, \ldots, k$, where $u_{0}=f$.

\section{Quasi-harmonic functions and Bergman spaces}

We introduce various spaces of functions on $M$ that will play an important role in what follows and investigate their mutual relations. Here $M$ is as in Section 2. Recall that $\mathscr{L}$ is an elliptic operator. Thus, given an open subset $\Omega$ of $M$, a positive integer $k$ and a constant $c$, every solution $u$ of the equation

$$
\mathscr{L}^{k} u=c \mathbf{1}_{\Omega}
$$

is smooth in $\Omega$.

The operator $\mathscr{L}$ has been defined in the introduction as the unique self-adjoint extension of minus the Laplace-Beltrami operator acting on $C_{c}^{\infty}(M)$. We recall that the domain of $\mathscr{L}$ in $L^{2}(M)$ is the space $\operatorname{Dom}(\mathscr{L})=\left\{u \in L^{2}(M): \mathscr{L} u \in L^{2}(M)\right\}$, where $\mathscr{L} u$ is interpreted in the sense of distributions [25]. Henceforth we shall also denote by $\mathscr{L}$ the natural extension of the Laplace-Beltrami operator to distributions.

Definition 3.1. Suppose that $k$ is a positive integer, and that $\Omega$ is a bounded open subset of $M$. We say that a function $v: \Omega \rightarrow \mathbb{C}$ is k-quasi-harmonic on $\Omega$ if $\mathscr{L}^{k} v$ is constant on $\Omega$ (in the sense of distributions, hence in the classical sense, since $v$ is smooth by elliptic regularity). We shall denote by $q_{k}^{2}(\Omega)$ the space of $k$-quasiharmonic functions on $\Omega$ which belong to $L^{2}(\Omega)$. The subspace of $q_{k}^{2}(\Omega)$ of all functions such that $\mathscr{L}^{k} v=0$ in $\Omega$ will be denoted by $h_{k}^{2}(\Omega)$ and will be called the ( $k^{\text {th }}$ generalised) Bergman space on $\Omega$.

Suppose now that $K$ is a compact subset of $M$. We say that $w: K \rightarrow \mathbb{C}$ is $k$-quasi-harmonic on $K$ if $w$ is the restriction to $K$ of a function in $q_{k}^{2}(\Omega)$, for some open set $\Omega$ containing $K$. We shall denote by $q_{k}^{2}(K)$ the space of all $k$ quasi-harmonic functions on $K$. The subspace of $q_{k}^{2}(K)$ of all functions which are restrictions to $K$ of functions in $h_{k}^{2}(\Omega)$ will be denoted by $h_{k}^{2}(K)$ and will be called the ( $k^{\text {th }}$ generalised) Bergman space on $K$.

Finally we shall denote by $q_{k}(M)$ the space of all $k$-quasi-harmonic functions on $M$. Notice that $q_{k}(M)$ is a space of functions in $C^{\infty}(M)$. 
Clearly $h_{k}^{2}(\Omega)$ is a subspace of $q_{k}^{2}(\Omega)$ of codimension one. Indeed, we have the vector space decomposition

$$
q_{k}^{2}(\Omega)=\left.h_{k}^{2}(\Omega) \oplus \mathbb{C}\left(\mathscr{L}^{-k} \mathbf{1}_{\Omega}\right)\right|_{\Omega} .
$$

Note that $\mathscr{L}^{-k} \mathbf{1}_{\Omega}$ is in $L^{2}(M)$, for the bottom $b$ of the spectrum of $\mathscr{L}$ is assumed to be positive, whence $\mathscr{L}^{-k}$ is bounded on $L^{2}(M)$.

Observe that both $q_{k}^{2}(\Omega)$ and $h_{k}^{2}(\Omega)$ are closed subspaces of $L^{2}(\Omega)$. Indeed, in view of the decomposition above it suffices to prove that $h_{k}^{2}(\Omega)$ is closed. Now, if $\left\{v_{n}\right\}$ is a sequence in $h_{k}^{2}(\Omega)$ that converges to $v$ in $L^{2}(\Omega)$, then $\mathscr{L}^{k} v_{n}$ tends to $\mathscr{L}^{k} v$ in the sense of distributions. Thus $\mathscr{L} v=0$, whence $v$ is in $h_{k}^{2}(\Omega)$. Clearly $q_{k}^{2}(\bar{\Omega})$ is contained in $q_{k}^{2}(\Omega)$. We shall prove below that if the boundary of $\Omega$ is smooth then $q_{k}^{2}(\bar{\Omega})$ is dense in $q_{k}^{2}(\Omega)$. To prove this, we need a few preliminary facts.

Definition 3.2. For a positive integer $m$ denote by $H^{m}(M)$ the Sobolev space of order $m$, i.e., the completion of

$$
\left\{u \in C^{\infty}(M): \nabla^{j} u \in L^{2}(M), j=0,1 \ldots, m\right\}
$$

with respect to the norm

$$
\|u\|_{H^{m}}:=\left(\sum_{j=0}^{m}\left\|\nabla^{j} u\right\|_{2}^{2}\right)^{1 / 2} .
$$

See [12] and the references therein for more on Sobolev spaces on manifolds.

Given a compact subset $K$ of $M$, denote by $H^{m}(M)_{K}$ the subspace of $H^{m}(M)$ of all functions whose support is contained in $K$, by $K$ the interior of $K$, and by $H_{0}^{m}(\stackrel{\circ}{K})$ the closure of $C_{c}^{\infty}(\stackrel{\circ}{K})$ in $H^{m}(M)$.

Suppose that $u$ is a function in $\operatorname{Dom}\left(\mathscr{L}^{k}\right)$ that vanishes in the complement of $K$. Then $\mathscr{L}^{k} u$ is in $L^{2}(M)$ and vanishes in $K^{c}$. By identifying $\mathscr{L}^{k} u$ with its restriction to $K$, we may interpret $\mathscr{L}^{k}$ as a map from $\operatorname{Dom}\left(\mathscr{L}^{k}\right)_{K}$ into $L^{2}(K)$. We shall make this identification in the sequel without further comment. Henceforth, if $E$ is a measurable subset of $M$ and $S$ is a subspace of $L^{2}(E)$, then we denote by $S^{\perp}$ its orthogonal in $L^{2}(E)$.

Lemma 3.3. Let $K$ be a compact subset of $M$. The following hold:

(i) $H^{2 k}(M)$ is contained in $\operatorname{Dom}\left(\mathscr{L}^{k}\right)$;

(ii) the map $\mathscr{L}^{k}$ is a Banach space isomorphism between $H^{2 k}(M)_{K}$ and $h_{k}^{2}(K)^{\perp}$ (the orthogonal complement of $h_{k}^{2}(K)$ in $L^{2}(K)$ ). 
Proof. First we prove (i). Let $T^{k} M$ be the bundle of covariant tensors of order $k$ and denote by $\operatorname{tr}: T^{k+2} M \rightarrow T^{k} M$ a trace, i.e. a metric contraction. Then for all sections $T$ of $T^{k+2} M$

$$
|\operatorname{tr}(T)(x)|_{x} \leq \sqrt{n}|T(x)|_{x} \quad \forall x \in M
$$

as can be easily seen by computing the trace in local coordinates given by an orthonormal frame and applying Schwarz's inequality.

Next we observe that the Laplacian $\mathscr{L}$ is bounded from the Sobolev space $H^{2 k+2}(M)$ to $H^{2 k}(M)$. Indeed if $u \in H^{2 k+2}(M)$ then, since the trace commutes with covariant derivatives,

$$
\nabla^{j} \mathscr{L} u=\nabla^{j} \operatorname{tr}\left(\nabla^{2} u\right)=\operatorname{tr}\left(\nabla^{j+2} u\right) \quad \forall j=0,1, \ldots, 2 k
$$

Thus

$$
\left\|\nabla^{j} \mathscr{L} u\right\|_{2} \leq \sqrt{n}\left\|\nabla^{j+2} u\right\|_{2}
$$

whence the boundedness of $\mathscr{L}$ from $H^{2 k+2}(M)$ to $H^{2 k}(M)$ follows.

To prove that $H^{2 k}(M) \subset \operatorname{Dom}\left(\mathscr{L}^{k}\right)$ we consider first the case $k=1$. If $u \in C^{\infty}(M) \cap H^{2}(M)$ then

$$
\|\mathscr{L} u\|_{2}=\left\|\operatorname{tr} \nabla^{2} u\right\|_{2} \leq \sqrt{n}\left\|\nabla^{2} u\right\|_{2} .
$$

The inclusion $H^{2}(M) \subset \operatorname{Dom}(\mathscr{L})$ follows, since $C^{\infty}(M) \cap H^{2}(M)$ is dense in $H^{2}(M)$ and $\mathscr{L}$ is closed. Finally, since $\operatorname{Dom}\left(\mathscr{L}^{k}\right)=\left\{u \in \operatorname{Dom}(\mathscr{L}): \mathscr{L} u \in \operatorname{Dom}\left(\mathscr{L}^{k-1}\right)\right\}$, the inclusion $H^{2 k}(M) \subset \operatorname{Dom}\left(\mathscr{L}^{k}\right)$ follows by induction on $k$.

Now we prove (ii). First we show that $\mathscr{L}^{k}$ maps $H^{2 k}(M)_{K}$ into $h_{k}^{2}(K)^{\perp}$. Suppose that $u$ is in $H^{2 k}(M)_{K}$ and $v$ is in $h_{k}^{2}(K)$. Denote by $\widetilde{v}$ a smooth function with compact support which is $k$-harmonic in an open neighbourhood of $K$ and satisfies $\widetilde{v}_{\left.\right|_{K}}=v$. Then

$$
\int_{K} v \mathscr{L}^{k} u \mathrm{~d} \mu=\int_{M} \tilde{v} \mathscr{L}^{k} u \mathrm{~d} \mu=\int_{M} \mathscr{L}^{k} \widetilde{v} u \mathrm{~d} \mu=0
$$

because the support of $u$ is contained in $K$ and $\mathscr{L}^{k} \widetilde{v}$ vanishes in a neighbourhood of $K$.

Since the bottom of the $L^{2}(M)$ spectrum of $\mathscr{L}$ is strictly positive, $\mathscr{L}^{k}$ is injective on its domain, hence on $H^{2 k}(M)_{K}$, for this is a subspace of $\operatorname{Dom}\left(\mathscr{L}^{k}\right)$ by (i) above.

Next we prove that $\mathscr{L}^{k}$ is onto. Suppose that $v$ is in $h_{k}^{2}(K)^{\perp}$. Denote by $\tilde{v}$ the extension of $v$ to a function on $M$ that vanishes off $K$. Set $u:=\mathscr{L}^{-k} \tilde{v}$. Clearly $u$ belongs to $\operatorname{Dom}\left(\mathscr{L}^{k}\right)$. We shall prove that $u$ is in $H^{2 k}(M)_{K}$. First we show that the 
support of $u$ is contained in $K$. For every smooth function $\phi$ with support contained in $K^{c}$

$$
\begin{aligned}
\int_{M} \phi u \mathrm{~d} \mu & =\int_{M} \mathscr{L}^{k} \mathscr{L}^{-k} \phi u \mathrm{~d} \mu \\
& =\int_{M} \mathscr{L}^{-k} \phi \mathscr{L}^{k} u \mathrm{~d} \mu=\int_{M} \mathscr{L}^{-k} \phi \tilde{v} \mathrm{~d} \mu=0 ;
\end{aligned}
$$

the last equality follows from the fact that $\mathscr{L}^{-k} \phi$ is $k$-harmonic in a neighbourhood of $K$ (hence its restriction to $K$ belongs to $\left.h_{k}^{2}(K)\right)$ and $v$ is in $h_{k}^{2}(K)^{\perp}$.

Since $\mathscr{L}^{k}$ is an elliptic operator of order $2 k$ and both $u$ and $\mathscr{L}^{k} u$ are functions in $L^{2}(M)$ with compact support, $u$ is in $H^{2 k}(M)$. Thus, $\mathscr{L}^{k}$ maps $H^{2 k}(M)_{K}$ onto $h_{k}^{2}(K)^{\perp}$ in a one-to-one fashion. Furthermore $\mathscr{L}^{k}$ is a continuous operator from $H^{2 k}(M)_{K}$ to $h_{k}^{2}(K)^{\perp}$. The closed graph theorem then implies that $\mathscr{L}^{-k}$ is continuous, thereby concluding the proof that $\mathscr{L}^{k}$ is a Banach space isomorphism.

Theorem 3.4. Let $K$ be a compact subset of $M$. The following are equivalent:

(i) $h_{k}^{2}(K)^{\perp}=h_{k}^{2}(\stackrel{\circ}{K})^{\perp}$;

(ii) $H^{2 k}(M)_{K}=H_{0}^{2 k}(\stackrel{\circ}{K})$.

Proof. First we prove that (i) implies (ii). Clearly $H_{0}^{2 k}(\stackrel{\circ}{K}) \subseteq H^{2 k}(M)_{K}$, so that it suffices to prove the inclusion $H^{2 k}(M)_{K} \subseteq H_{0}^{2 k}(\stackrel{\circ}{K})$, equivalently that $C_{c}^{\infty}(\stackrel{\circ}{K})$ is dense in $H^{2 k}(M)_{K}$. By the proof of Lemma 3.3, this is equivalent to the density of $\mathscr{L}^{k}\left(C_{c}^{\infty}(\stackrel{\circ}{K})\right)$ in $h_{k}^{2}(K)^{\perp}=h_{k}^{2}(\stackrel{\circ}{K})^{\perp}$, i.e., that the orthogonal space to $\mathscr{L}^{k}\left(C_{c}^{\infty}(\stackrel{\circ}{K})\right)$ in $h_{k}^{2}(\stackrel{\circ}{K})^{\perp}$ is the null space.

Suppose that $f$ is a function in $h_{k}^{2}(\stackrel{\circ}{K})^{\perp}$ that is orthogonal to $\mathscr{L}^{k} \phi$, for every $\phi$ in $C_{c}^{\infty}(\stackrel{\circ}{K})$. Denote by $\tilde{f}$ the extension of $f$ to a function on $M$ which vanishes in $(\stackrel{\circ}{K})^{c}$, and consider the distribution $\mathscr{L}^{k} \tilde{f}$. Then

$$
\left\langle\phi, \mathscr{L}^{k} \tilde{f}\right\rangle=\left\langle\mathscr{L}^{k} \phi, \tilde{f}\right\rangle=0 \quad \forall \phi \in C_{c}^{\infty}(\stackrel{\circ}{K}) .
$$

Thus, $\mathscr{L}^{k} \tilde{f}=0$ in $\stackrel{\circ}{K}$, so that $f$ belongs to $h_{k}^{2}(\stackrel{\circ}{K})$. But this implies that $f=0$ for $f$ is in $h_{k}^{2}(\stackrel{\circ}{K})^{\perp} \cap h_{k}^{2}(\stackrel{\circ}{K})$.

Next we prove that (ii) implies (i). Observe that the obvious inclusion $h_{k}^{2}(K) \subseteq$ $h_{k}^{2}(\stackrel{\circ}{K})$ implies the containment $h_{k}^{2}(\stackrel{\circ}{K})^{\perp} \subseteq h_{k}^{2}(K)^{\perp}$. Thus, it suffices to show that the assumption $H^{2 k}(M)_{K}=H_{0}^{2 k}(\stackrel{\circ}{K})$ implies

$$
h_{k}^{2}(K)^{\perp} \subseteq h_{k}^{2}(\stackrel{\circ}{K})^{\perp} .
$$

Suppose that $v$ is in $h_{k}^{2}(K)^{\perp}$. Write $\widetilde{v}$ for the extension of $v$ to $M$ which vanishes in $K^{c}$. By Lemma 3.3 there exists $u$ in $H^{2 k}(M)_{K}$ such that $\mathscr{L}^{k} u=\widetilde{v}$. The assumption 
$H^{2 k}(M)_{K}=H_{0}^{2 k}(\stackrel{\circ}{K})$ implies the existence of a sequence $\left\{\phi_{n}\right\}$ of functions in $C_{c}^{\infty}(\stackrel{\circ}{K})$ that is convergent to $u$ in $H^{2 k}(M)$. Then, if for every $f$ in $h_{k}^{2}\left(K_{K}^{\circ}\right)$ we denote by $\tilde{f}$ its extension to $M$ that vanishes off $\stackrel{\circ}{K}$,

$$
\begin{aligned}
\int_{\tilde{K}} v f \mathrm{~d} \mu & =\int_{M} \tilde{v} \tilde{f} \mathrm{~d} \mu=\int_{M} \mathscr{L}^{k} u \tilde{f} \mathrm{~d} \mu \\
& =\lim _{n \rightarrow \infty} \int_{M} \mathscr{L}^{k} \phi_{n} \tilde{f} \mathrm{~d} \mu=\lim _{n \rightarrow \infty}\left\langle\phi_{n}, \mathscr{L}^{k} \tilde{f}\right\rangle,
\end{aligned}
$$

which vanishes because the support of $\phi_{n}$ is contained in $\stackrel{\circ}{K}$ and $\mathscr{L}^{k} \widetilde{f}=0$ in $\stackrel{\circ}{K}$. Therefore $v$ is in $h_{k}^{2}\left(\AA^{\perp}\right)^{\perp}$, as required.

The above result raises the following question: which compact subsets $K$ of $M$ satisfy the requirement $H^{2 k}(M)_{K}=H_{0}^{2 k}(\stackrel{\circ}{K})$ ? In the case where $K=\overline{\stackrel{\circ}{K}}$ is a domain whose boundary is a smooth $(n-1)$-dimensional manifold, the spaces $H^{2 k}(M)_{K}$ and $H_{0}^{2 k}(\stackrel{\circ}{K})$ coincide by a well known result of Lions and Magenes (see [9, Theorem 2, page 259] for a proof in the Euclidean case, to which the result on $M$ can be reduced by passing to local coordinates via a partition of unity). More generally, we may use a version of the segment condition for manifolds (see [1, Theorem 5.29, page 159]). Unfortunately, this is not very useful in the setting of Riemannian manifolds, for the boundary of geodesic balls may contain even cusps (think of the elementary example of a cylinder in $\mathbb{R}^{3}$ ). However, it is a classical fact that if $r<\operatorname{Inj}_{p}$, then the boundary of $B(p, r)$ is a smooth $(n-1)$-dimensional submanifold of $M$, a fact that will be used without further comment in the sequel. Note that for every nonnegative integer $k$ and every open ball $B$, we have the orthogonal decompositions

$$
L^{2}(B)=q_{k}^{2}(B)^{\perp}+q_{k}^{2}(B)=q_{k}(\bar{B})^{\perp}+\overline{q_{k}^{2}(\bar{B})} .
$$

In fact, these decompositions coincide, at least for all $B$ such that $r_{B}<\operatorname{Inj}_{c_{B}}$, as the following result shows.

Proposition 3.5. Suppose that $B$ is an open ball in $M$, that $r_{B}<\operatorname{Inj}_{C_{B}}$ and that $k$ is a positive integer. The following hold:

(i) $\overline{q_{k}^{2}(\bar{B})}=q_{k}^{2}(B)$;

(ii) $q_{k}^{2}(B)$ is the set of all $v$ in $L^{2}(B)$ such that there exists a sequence $\left\{v_{n}\right\}$ of global $k$-quasi-harmonic functions such that

$$
\lim _{n \rightarrow \infty} \int_{B}\left|v-v_{n}\right|^{2} \mathrm{~d} \mu=0 .
$$

Proof. To prove (i) we first prove that if $v$ is in $\overline{q_{k}^{2}(\bar{B})}$, then $v$ is smooth on $B$ and $\mathscr{L}^{k} v$ is constant therein, i.e., $v$ is in $q_{k}^{2}(B)$. Indeed, there exists a sequence $\left\{v_{j}\right\}$ 
of functions in $C_{c}^{\infty}(M)$, such that $\mathscr{L}^{k} v_{j}$ is constant in a neighborhood of $\bar{B}$, that converges to $v$ in $L^{2}(B)$. Then $\left\{\mathscr{L}^{k} v_{j}\right\}$ converges to $\mathscr{L}^{k} v$ in $\mathscr{D}(B)$ so that $\mathscr{L}^{k} v$ is constant on $B$ in the sense of distributions, and, by elliptic regularity, $v$ is smooth on $B$, as required.

Conversely, suppose that $v$ is in $L^{2}(B)$ and that $\mathscr{L}^{k} v=c$ on $B$ in the sense of distributions for some constant $c$. Then $v$ is smooth in $B$ by elliptic regularity. Denote by $q_{0}$ a global $k$-quasi-harmonic function such that $\mathscr{L}^{k} q_{0}=1$ (such a function exists by Theorem 2.2 above). Then the function $v-c q_{0}$ is in the Bergman space $h_{k}^{2}(B)$. By Theorem 3.4 (i) and since $h_{k}^{2}(B)$ is closed in $L^{2}(B)$, the Bergman space $h_{k}^{2}(B)$ coincides with $\overline{h_{k}^{2}(\bar{B})}$. Hence there exists a sequence $\left\{h_{j}\right\}$ of $k$-harmonic functions in neighbourhoods of $\bar{B}$ such that

$$
\lim _{j \rightarrow \infty}\left\|v-c q_{0}-h_{j}\right\|_{L^{2}(B)}=0
$$

whence $\left\{h_{j}+c q_{0}\right\}$ converges to $v$ in $L^{2}(B)$, i.e., $v$ is in the closure of $q_{k}^{2}(\bar{B})$, as required.

Next we prove (ii). Clearly, if $V$ is a function in $L^{2}(B)$ that may be approximated in the $L^{2}(B)$-norm by a sequence of global $k$-quasi-harmonic functions, then it belongs to the closure of $q_{k}^{2}(\bar{B})$, which, by (i), is $q_{k}^{2}(B)$.

Conversely, suppose that $v$ is in $q_{k}^{2}(B)$. Then, by (i), it may be approximated in the $L^{2}$-norm by a sequence $\left\{u_{n}\right\}$ of $k$-quasi-harmonic functions in $q_{k}^{2}(\bar{B})$. Thus, it suffices to show that each of these functions may, in turn, be approximated in the $L^{2}(B)$-norm by global $k$-quasi-harmonic functions. Set $c_{n}:=\mathscr{L}^{k} u_{n}$. Denote by $q$ a global $k$-quasi-harmonic function such that $\mathscr{L}^{k} q=1$ on $M$. The function $u_{n}-c q$ is $k$ - harmonic in a neighbourhood of $\bar{B}$. Since $\bar{B}$ has no holes, there exists by Lemma 2.1 a global $k$-harmonic function $w_{n}$ such that

$$
\left|u_{n}-c q-w_{n}\right|<2^{-n} \quad \text { in } \bar{B} .
$$

The functions $v_{n}:=w_{n}+c q$ are the required approximants of $v$.

Remark 3.6. We note explicitly that if $M$ is a Cartan-Hadamard manifold, then

$$
\overline{q_{k}^{2}(\bar{B})}=q_{k}^{2}(B)
$$

for every geodesic ball $B$.

\section{Background on Hardy-type spaces}

Let $M$ denote a connected $n$-dimensional Riemannian manifold of infinite volume with Riemannian measure $\mu$. In this section we gather some known facts about the Hardy space $H^{1}(M)$, introduced by Carbonaro, Mauceri and Meda [5] in the 
setting of measured metric spaces of infinite volume (see also [6] for the case of finite volume), and the Hardy-type spaces $X^{k}(M)$, introduced in [16] and studied in [17].

Definition 4.1. We say that $M$ has $C^{\ell}$ bounded geometry if the injectivity radius is positive and the following hold:

- if $\ell=0$, then the Ricci tensor Ric is bounded from below;

- if $\ell$ is positive, then the covariant derivatives $\nabla^{j}$ Ric of the Ricci tensor are uniformly bounded on $M$ for all $j$ in $\{0, \ldots, \ell\}$.

Standing assumptions 4.2. Hereafter we make the following assumptions on $M$ :

(i) $b>0$ (b denotes the bottom of the $L^{2}$ - spectrum of $\left.\mathscr{L}\right)$;

(ii) $M$ has $C^{\ell}$ bounded geometry for some nonnegative integer $\ell$.

Remark 4.3. Set $\beta=\limsup \operatorname{su}_{r \rightarrow \infty}[\log \mu(B(o, r))] /(2 r)$, where $o$ is any reference point of $M$ and $B(o, r)$ denotes the geodesic ball with centre $o$ and radius $r$. By a result of Brooks $b \leq \beta^{2}$ [4]. It is well known that for manifolds with properties (i)-(ii) above there exist positive constants $\alpha$ and $C$ such that

$$
\mu(B(p, r)) \leq C r^{\alpha} \mathrm{e}^{2 \beta r} \quad \forall r \in[1, \infty) \quad \forall p \in M
$$

Furthermore [17, Remark 2.3] there exists a positive constant $C$ such that

$$
C^{-1} r^{n} \leq \mu(B(p, r)) \leq C r^{n} \quad \forall r \in(0,1] \quad \forall p \in M
$$

We denote by $\mathscr{B}$ the family of all balls on $M$. For each $B$ in $\mathscr{B}$ we denote by $c_{B}$ and $r_{B}$ the centre and the radius of $B$ respectively. Furthermore, we denote by $c B$ the ball with centre $c_{B}$ and radius $c r_{B}$. For each scale parameter $s$ in $\mathbb{R}^{+}$, we denote by $\mathscr{B}_{s}$ the family of all balls $B$ in $\mathscr{B}$ such that $r_{B} \leq s$.

We recall the definitions of the atomic Hardy space $H^{1}(M)$ and its dual space $\operatorname{BMO}(M)$ given in [5]. We set $s_{0}:=(1 / 2) \operatorname{Inj}(M)$.

Definition 4.4. An $H^{1}$-atom $a$ is a function in $L^{2}(M)$ supported in a ball $B$ with the following properties:

(i) $\int_{B} a \mathrm{~d} \mu=0$;
(ii) $\|a\|_{2} \leq \mu(B)^{-1 / 2}$.

Given a positive "scale parameter" $s$, we say that an $H^{1}$-atom $a$ is at scale $s$ if it is supported in a ball $b$ of $\mathscr{B}_{s}$. An $H^{1}$-atom is called admissible if it is supported in a ball $B$ of $\mathscr{B}_{s_{0}}$. 
Definition 4.5. The Hardy space $H^{1}(M)$ is the space of all functions $f$ in $L^{1}(M)$ that admit a decomposition of the form

$$
f=\sum_{j=1}^{\infty} c_{j} a_{j}
$$

where $a_{j}$ are admissible $H^{1}$-atoms, and $\sum_{j=1}^{\infty}\left|c_{j}\right|<\infty$. The norm $\|f\|_{H^{1}}$ of $f$ is the infimum of $\sum_{j=1}^{\infty}\left|c_{j}\right|$ over all decompositions (4.3) of $f$.

We denote with $H_{\text {fin }}^{1}(M)$ the vector space of all finite linear combinations of admissible $H^{1}$-atoms, endowed with the norm

$$
\|f\|_{H_{\text {fin }}^{1}}=\inf \left\{\sum_{j=1}^{N}\left|c_{j}\right|: f=\sum_{j=1}^{N} c_{j} a_{j}, a_{j} \text { admissible } H^{1} \text {-atom, } N \in \mathbb{N}\right\} .
$$

It is known that, under the Standing assumptions 4.2, the $H^{1}$-norm and the $H_{\text {fin }}^{1}$ norm are equivalent on $H_{\text {fin }}^{1}(M)$ [15, Section 4].

Remark 4.6. Actually, in the definition of the spaces $H^{1}(M)$ and $H_{\text {fin }}^{1}(M)$, the choice of scale is irrelevant. Indeed, in [5] it has been shown that in Definition 4.5 one obtains the same spaces, with equivalent norms, if admissible atoms are replaced by atoms at any fixed scale $s$.

Definition 4.7. We define $\operatorname{BMO}(M)$ as the space of all locally integrable functions $g$ such that

$$
\|g\|_{\mathrm{BMO}}:=\sup _{B \in \mathscr{B}_{s_{0}}} \inf _{c \in \mathbb{C}}\left(\frac{1}{\mu(B)} \int_{B}|g-c|^{2} \mathrm{~d} \mu\right)^{1 / 2}<\infty .
$$

The Banach dual of $H^{1}(M)$ is isomorphic to $\operatorname{BMO}(M) / \mathbb{C}$ [5, Theorem 5.1].

Now we recall the definition of the generalised Hardy spaces $X^{k}(M)$. For $\sigma>0$ denote by $\mathscr{U}_{\sigma}$ the operator $\mathscr{L}(\sigma \mathscr{I}+\mathscr{L})^{-1}$. It is known that for every positive integer $k$ the operator $\mathscr{U}_{\sigma}^{k}$ is injective on $L^{1}(M)+L^{2}(M)$ [16, Proposition 2.4 (ii)].

Definition 4.8. For each positive integer $k$ and for each $\sigma>\beta^{2}-b$ we denote by $X^{k}(M)$ the Banach space of all $L^{1}(M)$ functions $f$ such that $\mathscr{U}_{\sigma}^{-k} f$ is in $H^{1}(M)$, endowed with the norm

$$
\|f\|_{X^{k}}=\left\|\mathscr{U}_{\sigma}^{-k} f\right\|_{H^{1}} .
$$

Clearly $\mathscr{U}_{\sigma}^{-k}$ is an isometric isomorphism between $X^{k}(M)$ and $H^{1}(M)$. It is known [16, Section 3] that the space $X^{k}(M)$ does not depend on $\sigma>\beta^{2}-b$, and that different values of $\sigma$ give rise to equivalent norms on $X^{k}(M)$. For later use, it is convenient to assume that $\sigma>2 \beta$, and we shall denote $\mathscr{U}_{\sigma}$ simply by $\mathscr{U}$. 
Definition 4.9. For each positive integer $k$ we denote by $Y^{k}(M)$ the Banach dual of $X^{k}(M)$.

Remark 4.10. Since $\mathscr{U}^{-k}$ is an isometric isomorphism between $X^{k}(M)$ and $H^{1}(M)$, the transpose map $\left(\mathscr{U}^{-k}\right)^{t}$ is an isometric isomorphism between the dual of $H^{1}(M)$, i.e., $\mathrm{BMO}(M) / \mathbb{C}$, and $Y^{k}(M)$. Hence

$$
\left\|\left(\mathscr{U}^{-k}\right)^{t} f\right\|_{Y^{k}}=\|f\|_{\mathrm{BMO} / \mathbb{C}} .
$$

Some properties of $X^{k}(M)$ are listed in the introduction (see [16,17]). The space $X^{k}(M)$ admits an atomic decomposition in terms of "special atoms" [17], which we now define.

Definition 4.11. Let $k$ be a positive integer and $B$ a ball in $M$. An $X^{k}$-atom associated to the ball $B$ is a function $A$ in $L^{2}(M)$, supported in $B$, such that:

(i) $\int A h \mathrm{~d} \mu=0 \quad \forall h \in q_{k}^{2}(\bar{B})$;

(ii) $\|A\|_{2} \leq \mu(B)^{-1 / 2}$.

Note that condition (i) implies that $\int_{M} A \mathrm{~d} \mu=0$, because $\mathbf{1}_{2 B}$ is in $q_{k}^{2}(\bar{B})$. Given a positive "scale parameter" $s$, we say that an $X^{k}$-atom is at scale $s$ if it is supported in a ball $B$ of $\mathscr{B}_{s}$. As in the case of $H^{1}$, atoms at scale $s_{0}$ will simply be called admissible $X^{k}$-atoms.

Observe that $X^{k}$-atoms satisfy an infinite dimensional cancellation condition. In [17] we proved the following result.

Theorem 4.12. Suppose that $k$ is a positive integer and that $M$ has $C^{2 k-2}$ bounded geometry (see Definition 4.1). Choose a "scale parameter" $s$. Then the space $X^{k}(M)$ is the space of all functions $F$ in $H^{1}(M)$ that admit a decomposition of the form $F=\sum_{j} c_{j} A_{j}$, where $\left\{c_{j}\right\}$ is a sequence in $\ell^{1}$ and $\left\{A_{j}\right\}$ is a sequence of admissible $X^{k}$-atoms at scale s. Furthermore

$\|F\|_{X^{k}} \asymp \inf \left\{\sum_{j}\left|c_{j}\right|: F=\sum_{j} c_{j} A_{j}, \quad\right.$ where $A_{j}$ are $X^{k}$-atoms at scale $\left.s\right\}$.

Notice that the equivalence of norms above implies that "atomic norms" associated to different "scale parameters" $s_{1}$ and $s_{2}$ are equivalent on $X^{k}(M)$. As in the definition of $H^{1}(M)$, a convenient choice of the scale parameter is $s_{0}:=(1 / 2) \operatorname{Inj}(M)$. This choice of the scale parameter will simplify most of the arguments below, for balls of radius at most $s_{0}$ have no holes and their boundaries are smooth, whence the theory developed in Sections 3 and 4 applies. In particular, in view of Proposition 3.5 the cancellation condition of an $X^{k}$-atom $A$ associated to a ball $B$ may be described in one of the following equivalent ways:

(a) $\quad \int_{B} A v \mathrm{~d} \mu=0 \quad \forall v \in q_{k}^{2}(\bar{B})$; 
$\begin{array}{ll}\text { (b) } \quad \int_{B} A v \mathrm{~d} \mu=0 & \forall v \in q_{k}^{2}(B) \text {; } \\ \text { (c) } \quad \int_{B} A v \mathrm{~d} \mu=0 & \forall v \in q_{k}(M) \text {. }\end{array}$

We remark also that $X^{1}(M)$ admits an atomic decomposition in terms of $X^{1}$-atoms whenever $M$ satisfies mild geometric assumptions, i.e. $M$ has positive injectivity radius, Ricci curvature bounded from below and spectral gap, whereas if $k \geq 2$, then the atomic decomposition of $X^{k}(M)$ requires at least $C^{2}$ bounded geometry.

Next we introduce a norm on the space of finite linear combinations of admissible $X^{k}$-atoms.

Definition 4.13. Suppose that $k$ is a positive integer. We denote by $X_{\text {fin }}^{k}(M)$ the vector space of all finite linear combinations of admissible $X^{k}$-atoms, endowed with the norm

$$
\|F\|_{X_{\text {fin }}^{k}}:=\inf \left\{\sum_{j=1}^{N}\left|c_{j}\right|: F=\sum_{j=1}^{N} c_{j} A_{j}, A_{j} \text { admissible } X^{k} \text {-atom }\right\} .
$$

Remark 4.14. By combining [17, Remark 3.5] with the proof of [17, Lemma 6.1] one can see that any $X^{k}$-atom $A$ at scale $s>s_{0}$ can be written as a finite linear combination $A=\sum_{j=1}^{N(s)} \lambda_{j} A_{j}$ of admissible $X^{k}$-atoms $A_{j}$, with $\sum\left|\lambda_{j}\right| \leq C s$. Thus, if in the definition of $X_{\text {fin }}^{k}(M)$ we replace admissible $X^{k}$-atom by $X^{k}$-atoms at any fixed scale $s$, we obtain the same space with an equivalent norm.

Remark 4.15. Notice that $X_{\text {fin }}^{k}(M)$ is contained in $\mathscr{U}^{k}\left(H_{\text {fin }}^{1}(M)\right)$. Indeed, for any admissible $X^{k}$-atom $A, \mathscr{U}^{-k} A$ is a multiple of an admissible $H^{1}$-atom by [17, Remark 3.5]. Hence, $\mathscr{U}^{-k} A$ lies in $H_{\text {fin }}^{1}(M)$. It follows that $A=\mathscr{U}^{k} \mathscr{U}^{-k} A$ belongs to $\mathscr{U}^{k}\left(H_{\text {fin }}^{1}(M)\right)$.

Clearly

$$
\|F\|_{X^{k}} \leq\|F\|_{X_{\text {fin }}^{k}} \quad \forall F \in X_{\text {fin }}^{k}(M),
$$

so that there is a natural injection of the completion of $X_{\text {fin }}^{k}(M)$ into $X^{k}(M)$. We shall show that this map is an isomorphism of Banach spaces.

Lemma 4.16. Suppose that $k$ is a positive integer and that $M$ has $C^{2 k-2}$ bounded geometry. If a is an $H^{1}$-atom in $\operatorname{Dom}\left(\mathscr{L}^{k}\right)$, then $\mathscr{L}^{k}$ a is in $X_{\text {fin }}^{k}(M)$. Furthermore, if the support of $a$ is contained in the ball $B$, then there exists a constant $C$ such that

$$
\left\|\mathscr{L}^{k} a\right\|_{X_{\text {fin }}^{k}} \leq C\left(1+r_{B}\right) \mu(B)^{1 / 2}\left\|\mathscr{L}^{k} a\right\|_{2} .
$$

Proof. See [17, Lemma 6.1], where the statement is proved for the norm in $X^{k}(M)$ instead of $X_{\text {fin }}^{k}(M)$. It is straightforward to check that the proof of [17, Lemma 6.1] proves the stronger statement above. 
In the following lemma and elsewhere we shall identify functions in $q_{k}^{2}(B)^{\perp}$ with their extensions to $M$ that vanish outside $B$.

Lemma 4.17. Suppose that $k$ is a positive integer and that $M$ has $C^{2 k-2}$ bounded geometry. There exists a constant $C$ such that for every ball $B$ and every $F$ in $q_{k}^{2}(B)^{\perp}$

$$
\|F\|_{X_{\text {fin }}^{k}} \leq C\left(1+r_{B}\right)\left(\mu(B)^{1 / 2}\|F\|_{2}\right) .
$$

Proof. The function $A:=F /\left(\mu(B)^{1 / 2}\|F\|_{2}\right)$ is an $X^{k}$ - atom with support contained in $\bar{B}$. Hence $a:=\mathscr{L}^{-k} A /\left\|\mathscr{L}^{-k}\right\|_{2}$ is a $H^{1}$-atom with support contained in $\bar{B}$. Note that $a$ is in $\operatorname{Dom}\left(\mathscr{L}^{k}\right)$. By Lemma 4.16

$$
\left\|\mathscr{L}^{k} a\right\|_{X_{\text {fin }}^{k}} \leq C\left(1+r_{B}\right) \mu(B)^{1 / 2}\left\|\mathscr{L}^{k} a\right\|_{2},
$$

from which the required estimate follows directly.

We also need the following result, which provides a "nice" decomposition of $\mathscr{U}^{k} a$ for an admissible $H^{1}$-atom $a$ in terms of admissible $X^{k}$-atoms.

Lemma 4.18. Suppose that $k$ is a positive integer and that $M$ has $C^{2 k-2}$ bounded geometry. Let a be an admissible $H^{1}$-atom supported in a ball $B(p, R)$, where $p$ is in $M$ and $R \leq s_{0}$. Then there exist a positive constant $C$, functions $A_{i}^{\prime}$ and $A_{j}^{\prime \prime}$ such that

$$
\mathscr{U}^{k} a=\sum_{i=0}^{d} A_{i}^{\prime}+\sum_{j=1}^{\infty} A_{j}^{\prime \prime},
$$

where $d=\left[\log _{4}(3 / R)+1\right]$, the series converges in $X^{k}(M)$ and in $L^{2}(M)$, and

(i) the function $A_{i}^{\prime}$ is supported in $B_{i}^{\prime}=B\left(p,\left(4^{i}+1\right) R\right)$, lies in $q_{k}^{2}\left(B_{i}^{\prime}\right)^{\perp}$ and

$$
\left\|A_{i}^{\prime}\right\|_{2} \leq C \mu\left(B_{i}^{\prime}\right)^{-1 / 2} 4^{-i}
$$

(ii) the function $A_{j}^{\prime \prime}$ is supported in $B_{j}^{\prime \prime}=B(p, j+1)$, lies in $q_{k}^{2}\left(B_{j}^{\prime \prime}\right)^{\perp}$ and

$$
\left\|A_{j}^{\prime \prime}\right\|_{2} \leq C \mathrm{e}^{-2 \beta j}
$$

Moreover, $A_{j}^{\prime \prime}$ lies in $X_{\text {fin }}^{k}(M)$ and there exist positive constants $C$ and $\varepsilon$, such that

$$
\left\|A_{j}^{\prime \prime}\right\|_{X_{\text {fin }}^{k}} \leq C \mathrm{e}^{-\varepsilon j} .
$$


Proof. Since the proof is a slight variation of the argument used in the proof of [17, Lemma 4.2], we explain only the differences between the two arguments. Let $\mathscr{D}_{1}=\sqrt{\mathscr{L}-b+\kappa^{2}}$, where $-\kappa^{2}$ is a lower bound for the Ricci curvature, and $\mathscr{R}=r\left(\mathscr{D}_{1}\right)$, where $r(t)=\frac{1}{c^{2}+t^{2}}$ with $c^{2}=4 \beta^{2}+b$. Write

$$
\mathscr{U}^{k} a=\mathscr{L}^{k} \mathscr{R}^{k} a=\mathscr{L}^{k} \mathscr{S} a+\mathscr{L}^{k} \mathscr{T} a,
$$

where $\mathscr{S}$ and $\mathscr{T}$ are the operators defined spectrally as in equation (6.14) of [17], with $r$ replaced by $r^{k}$. Then, as in the proof of [17, Lemma 4.2], one sees that

$$
\mathscr{S}=\sum_{i=0}^{d} S_{i}\left(\mathscr{D}_{1}\right), \quad \mathscr{T}=\sum_{j=1}^{\infty} T_{j}\left(\mathscr{D}_{1}\right),
$$

where the functions $S_{i}$ and $T_{j}$ are defined as in equation (6.9), (6.10) and (6.23) of [17], where again $r$ has been replaced by $r^{k}$. To conclude the proof it suffices to define

$$
A_{i}^{\prime}=\mathscr{L}^{k} S_{i}\left(\mathscr{D}_{1}\right) a, \quad A_{j}^{\prime \prime}=\mathscr{L}^{k} T_{j}\left(\mathscr{D}_{1}\right) a,
$$

and argue as in the proof of [17, Lemma 4.2].

\section{The dual of Hardy-type spaces}

In this section we prove our main result, which identifies the dual of $X^{k}(M)$ to a Banach space of functions on $M$. We need more notation and some preliminary results. For any open ball $B$ in $M$, we denote by $\tilde{\pi}_{B, k}: L^{2}(B) \rightarrow q_{k}^{2}(B)^{\perp}$ the orthogonal projection onto $q_{k}^{2}(B)^{\perp}$. We may extend $\tilde{\pi}_{B, k}$ to a map $\pi_{B, k}$ from $L_{\text {loc }}^{2}(M)$ to $q_{k}^{2}(B)^{\perp}$, by setting

$$
\pi_{B, k}(F):=\tilde{\pi}_{B, k}\left(\left.F\right|_{B}\right) \quad \forall F \in L_{\mathrm{loc}}^{2}(M),
$$

where $\left.F\right|_{B}$ denotes the restriction of $F$ to $B$.

Proposition 5.1. Suppose that $s>0, G$ is in $L_{\mathrm{loc}}^{2}(M)$ and $\pi_{B, k}(G)=0$ for every $B$ in $\mathscr{B}_{s}$. Then $G$ is a global $k$-quasi-harmonic function, i.e. it belongs to $q_{k}(M)$.

Proof. Observe that $\pi_{B, k}(G)=\tilde{\pi}_{B, k}\left(\left.G\right|_{B}\right)$. Hence, $\left.G\right|_{B}$ is orthogonal to $q_{k}^{2}(B)^{\perp}$, i.e., it belongs to $q_{k}^{2}(B)$. Thus, $\mathscr{L}^{k} G$ is constant on $B$. Now, if $B$ and $B^{\prime}$ are two balls in $\mathscr{B}_{s}$ with nonempty intersection, $\mathscr{L}^{k} G$ is constant both on $B$ and on $B^{\prime}$, whence the constant must be the same for the two balls. Since $M$ is connected by assumption, $\mathscr{L}^{k} G$ is constant on $M$, i.e. it is in $q_{k}(M)$, as required.

Definition 5.2. Suppose that $k$ is a positive integer and $s>0$. Then $\operatorname{GBMO}_{s}^{k}(M)$ is the vector space of all functions $G$ in $L_{\text {loc }}^{2}(M)$ such that

$$
\|G\|_{\mathrm{GBMO}_{s}^{k}}:=\sup _{B \in \mathscr{B}_{s}} \mu(B)^{-1 / 2}\left\|\pi_{B, k}(G)\right\|_{2}<\infty .
$$


Note that if $r_{B}<\operatorname{Inj}(M)$, then by Proposition 3.5 (ii)

$$
\left\|\pi_{B, k}(G)\right\|_{2}=\inf _{V \in q_{k}(M)}\left[\int_{B}|G-V|^{2} \mathrm{~d} \mu\right]^{1 / 2} .
$$

Loosely speaking, if $s<\operatorname{Inj}(M)$, then the space $\operatorname{GBMO}_{s}^{k}(M)$ consists of all locally square-integrable functions $G$, which are "well approximated" on each ball $B$ in $\mathscr{B}_{S}$ by global $k$-quasi-harmonic functions. If we interpret constants as 0 quasi-harmonic functions, we may say that $\operatorname{BMO}(M)$ functions are those locally square-integrable functions, which are "well approximated" on each ball $B$ in $\mathscr{B}_{s}$ by 0 -quasi-harmonic functions. Thus, functions in $\mathrm{GBMO}_{s}^{k}(M)$ may be considered as generalisations of functions in $\operatorname{BMO}(M)$, a fact which partially justifies the notation.

Henceforth, we shall consider the spaces $\operatorname{GBMO}_{s}^{k}(M)$ only for $s<\operatorname{Inj}(M)$ and we shall write $\mathrm{GBMO}^{k}(M)$ instead of $\operatorname{GBMO}_{s_{0}}^{k}(M)$, where $s_{0}=\frac{1}{2} \operatorname{Inj}(M)$. We shall prove later that if $s$ is less than $\operatorname{Inj}(M)$ then the spaces $\mathrm{GBMO}_{s}^{k}(M)$ do not depend on $s$ and that all the norms $\|\cdot\|_{\mathrm{GBMO}_{s}^{k}}, 0<s<\operatorname{Inj}(M)$, are equivalent (see Corollary 5.9).

Obviously, $\|\cdot\|_{\mathrm{GBMO}_{s}^{k}}$ vanishes on $q_{k}(M)$ and, by Proposition 5.1, it defines a norm on the quotient space $\operatorname{GBMO}_{s}^{k}(M) / q_{k}(M)$. Note that if $k \leq \ell$ then a function $G$ in $\operatorname{GBMO}_{s}^{k}(M)$ is also in $\mathrm{GBMO}_{s}^{\ell}(M)$, for

$$
\left\|\pi_{B, \ell}(G)\right\|_{2} \leq\left\|\pi_{B, k}(G)\right\|_{2} .
$$

In particular, any representative of a class in $\operatorname{BMO}(M) / \mathbb{C}$, represents also a class in $\mathrm{GBMO}_{s}^{\ell}(M) / q_{\ell}(M)$.

The main result of this section (Theorem 5.8 below) is that the dual $Y^{k}(M)$ of $X^{k}(M)$ can be identified with $\mathrm{GBMO}^{k}(M) / q_{k}(M)$ via the map $\iota$ that to each coset $G+q_{k}(M)$ in $\mathrm{GBMO}^{k}(M) / q_{k}(M)$ associates the functional $\iota\left(G+q_{k}(M)\right)$ on $X_{\text {fin }}^{k}(M)$ defined by

$$
\iota\left(G+q_{k}(M)\right)(F):=\int_{M} F G \mathrm{~d} \mu \quad \forall F \in X_{\text {fin }}^{k}(M) .
$$

It is straightforward to check that the integral above does not change if we replace $G$ by any other representative of the coset $G+q_{k}(M)$. At this point it is by no means clear that the functional $\iota\left(G+q_{k}(M)\right)$ extends to a continuous linear functional on $X^{k}(M)$. We shall prove that this is indeed the case and that $\iota$ extends to a Banach space isomorphism between $\mathrm{GBMO}^{k}(M) / q_{k}(M)$ and $Y^{k}(M)$ (see Theorem 5.8).

To prove this result it is useful to introduce another space that will play also a role in the characterization of the dual of $X_{\text {fin }}^{k}(M)$ in the next section.

Definition 5.3. Suppose that $k$ is a positive integer. We define $\mathbb{Y}^{k}(M)$ to be the space of all families of functions $\mathbf{G}:=\left\{G_{B}: B \in \mathscr{B}\right\}$ such that 
(i) $G_{B}$ is in $q_{k}^{2}(B)^{\perp}$ and $\pi_{B, k}\left(G_{B^{\prime}}\right)=G_{B}$ for all $B, B^{\prime} \in \mathscr{B}$ such that $B \subset B^{\prime}$;

(ii) $\|\mathbf{G}\|_{\mathbb{Y}^{k}}:=\sup _{B \in \mathscr{B}_{s_{0}}}\left(\frac{1}{\mu(B)} \int_{B}\left|G_{B}\right|^{2} \mathrm{~d} \mu\right)^{1 / 2}<\infty$.

It is straightforward to check that if $G$ is in $\operatorname{GBMO}^{k}(M)$ then the collection $\mathbf{G}:=$ $\left\{\pi_{B, k}(G): B \in \mathscr{B}\right\}$ is in $\mathbb{Y}^{k}(M)$, and

$$
\|\mathbf{G}\|_{\mathbb{Y}^{k}}=\|G\|_{\mathrm{GBMO}^{k}} .
$$

Conversely, given $\mathbf{G}=\left\{G_{B}: B \in \mathscr{B}\right\}$ in $\mathbb{Y}^{k}(M)$, it is not clear a priori whether there exists $G$ in $\mathrm{GBMO}^{k}(M)$ such that $G_{B}=\pi_{B, k}(G)$ for every $B$ in $\mathscr{B}$. In Corollary 5.11 we shall prove that this is indeed the case, following a somewhat long detour. It would be nice to have a more direct proof of this fact.

Definition 5.4. Given a function $h: \mathscr{B} \rightarrow \mathbb{C}$ and a complex number $\alpha$, we say that $\lim _{B} h(B)=\alpha$ if for every $\varepsilon>0$ there exists a ball $B_{\varepsilon}$ such that

$$
|h(B)-\alpha|<\varepsilon \quad \forall B \in \mathscr{B} \text { such that } B_{\varepsilon} \subset B .
$$

Fix a reference point $o$ in $M$, and, for every positive integer $m$, denote by $B_{m}$ the ball with centre $o$ and radius $m$. It is straightforward to check that if $h: \mathscr{B} \rightarrow \mathbb{C}$ and $\lim _{B} h(B)=\alpha \in \mathbb{C}$, then $\lim _{m \rightarrow \infty} h\left(B_{m}\right)=\alpha$.

Lemma 5.5. Suppose that $k$ is a positive integer and that $M$ has $C^{2 k-2}$ bounded geometry. The following hold:

(i) for every $\mathbf{G}$ in $\mathbb{Y}^{k}(M)$ the linear functional $\lambda_{\mathbf{G}}$ on $X_{\text {fin }}^{k}(M)$, defined by

$$
\lambda_{\mathbf{G}}(F)=\lim _{B} \int_{M} F G_{B} \mathrm{~d} \mu \quad \forall F \in X_{\text {fin }}^{k}(M),
$$

is well defined and it is continuous on $X_{\text {fin }}^{k}(M)$ and $\left\|\lambda_{\mathbf{G}}\right\|_{\left(X_{\text {fin }}^{k}\right)^{*}} \leq\|\mathbf{G}\|_{\mathbb{Y}^{k}}$;

(ii) there exists a positive constant $C$ such that for every $B$ in $\mathscr{B}$ and for every $\mathbf{G}$ in $\mathbb{Y}^{k}(M)$

$$
\left(\frac{1}{\mu(B)} \int_{B}\left|G_{B}\right|^{2} \mathrm{~d} \mu\right)^{1 / 2} \leq C\|\mathbf{G}\|_{\mathbb{Y}^{k}}\left(1+r_{B}\right) ;
$$

(iii) for every admissible $H^{1}$-atom a and for every $\mathbf{G}$ in $\mathbb{Y}^{k}(M)$, the limit

$$
\lambda\left(\mathscr{U}^{k} a\right):=\lim _{m \rightarrow \infty}\left(\mathscr{U}^{k} a, G_{B_{m}}\right)
$$

exists and does depend on the reference point $o$. Furthermore, there exists a positive constant $C$ such that

$$
\left|\lambda\left(\mathscr{U}^{k} a\right)\right| \leq C\|\mathbf{G}\|_{\mathbb{Y}^{k}} ;
$$


(iv) for every $\mathbf{G}$ in $\mathbb{Y}^{k}(M)$ the linear functional $\lambda_{\mathbf{G}}$ on $X_{\text {fin }}^{k}(M)$, defined in (i), extends to a continuous linear functional on $X^{k}(M)$, and there exists a constant $C$, independent of $\mathbf{G}$, such that $\left\|\lambda_{\mathbf{G}}\right\|_{\left(X^{k}\right)^{*}} \leq C\|\mathbf{G}\|_{\mathbb{Y}^{k}}$;

(v) for every $G$ in $\mathrm{GBMO}^{k}(M)$ the linear functional $\iota\left(G+q_{k}(M)\right)$ on $X_{\text {fin }}^{k}(M)$, defined by

$$
\iota\left(G+q_{k}(M)\right)(F)=\int_{M} F G \mathrm{~d} \mu \quad \forall F \in X_{\text {fin }}^{k}(M),
$$

extends to a unique continuous linear functional on $X^{k}(M)$, and the map ı : $\mathrm{GBMO}^{k}(M) / q_{k}(M) \rightarrow Y^{k}(M)$ that associates to the coset $G+q_{k}(M)$ the extension of $\iota\left(G+q_{k}(M)\right)$ described above is a continuous linear map.

Proof. First we prove (i). Note that the limit

$$
\lim _{B} \int_{M} F G_{B} \mathrm{~d} \mu \quad \forall F \in X_{\text {fin }}^{k}(M)
$$

exists, for the support of $F$ is contained in a ball. Thus, $\lambda_{\mathbf{G}}(F)$ is well defined. Suppose that $A$ is an $X^{k}$-atom with support contained in a ball $B$ in $\mathscr{B}_{s_{0}}$. We have that

$$
\begin{aligned}
\left|\lambda_{\mathbf{G}}(A)\right| & =\left|\int_{B} A G_{B} \mathrm{~d} \mu\right| \\
& \leq\|A\|_{2}\left\|G_{B}\right\|_{2} \\
& \leq \mu(B)^{-1 / 2}\left\|G_{B}\right\|_{2} \\
& \leq\|\mathbf{G}\|_{\mathbb{Y}^{k}} .
\end{aligned}
$$

Therefore, if $F=\sum_{j=1}^{N} c_{j} A_{j}$ is in $X_{\text {fin }}^{k}(M)$, then

$$
\left|\lambda_{\mathbf{G}}(F)\right| \leq\|\mathbf{G}\|_{\mathbb{Y}^{k}} \sum_{j=1}^{N}\left|c_{j}\right| .
$$

We now take the infimum of both sides with respect to all finite representations of $F$, and obtain

$$
\left|\lambda_{\mathbf{G}}(F)\right| \leq\|\mathbf{G}\|_{\mathbb{Y}^{k}}\|F\|_{X_{\text {fin }}^{k}} \quad \forall F \in X_{\text {fin }}^{k}(M),
$$

as required to conclude the proof of (i).

Next we prove (ii). If $r_{B} \leq s_{0}$, then the required estimate follows directly from the definition of the space $\mathbb{Y}^{k}(M)$.

Suppose that $r_{B}>s_{0}$. Denote by $\lambda_{\mathbf{G}}$ the continuous linear functional on $X_{\text {fin }}^{k}(M)$ associated to $\mathbf{G}$ as in (i). Then

$$
\begin{aligned}
\left|\left(F, G_{B}\right)\right| & =\left|\lambda_{\mathbf{G}}(F)\right| \\
& \leq\left\|\lambda_{\mathbf{G}}\right\|_{\left(X_{\text {fin }}^{k}\right)^{*}}\|F\|_{X_{\text {fin }}^{k}} \\
& \leq C\left\|\lambda_{\mathbf{G}}\right\|_{\left(X_{\text {fin }}^{k}\right)^{*}}\left(1+r_{B}\right) \mu(B)^{1 / 2}\|F\|_{2} \quad \forall F \in q_{k}^{2}(B)^{\perp},
\end{aligned}
$$


by Lemma 4.17 . By taking the supremum of both sides over all $F$ in $q_{k}^{2}(B)^{\perp}$ such that $\|F\|_{2}=1$, we obtain that

$$
\left\|G_{B}\right\|_{2} \leq C\left\|\lambda_{\mathbf{G}}\right\|_{\left(X_{\text {fin }}^{k}\right)^{*}}\left(1+r_{B}\right) \mu(B)^{1 / 2},
$$

which, in view of (i), implies the required conclusion.

Now, we prove (iii). Suppose that the atom $a$ is supported in the ball $B(p, R)$ with $R \leq s_{0}$. Observe preliminarily that both $\mathscr{U}^{k} a$ and $G_{B_{m}}$ (recall that $B_{m}$ denotes the ball with centre $o$ and radius $m$ ) are in $L^{2}(M)$, so that the inner product $\left(\mathscr{U}^{k} a, G_{B_{m}}\right)$ in the statement makes sense. By Lemma 4.18 , we may write

$$
\mathscr{U}^{k} a=\sum_{i=0}^{d} A_{i}^{\prime}+\sum_{j=1}^{\infty} A_{j}^{\prime \prime}
$$

where the series $\sum_{j=1}^{\infty} A_{j}^{\prime \prime}$ converges in $L^{2}(M), A_{i}^{\prime}$ is supported in $B_{i}^{\prime}$ and $A_{i}^{\prime \prime}$ is supported in $B_{i}^{\prime \prime}$. Therefore

$$
\left(\mathscr{U}^{k} a, G_{B_{m}}\right)=\sum_{i=1}^{d}\left(A_{i}^{\prime}, G_{B_{m}}\right)+\sum_{j=1}^{\infty}\left(A_{j}^{\prime \prime}, G_{B_{m}}\right) .
$$

By (i) for every $1 \leq i \leq d$ and for every $j \geq 1$ the limits $\lim _{m \rightarrow \infty}\left(A_{i}^{\prime}, G_{B_{m}}\right)$ and $\lim _{m \rightarrow \infty}\left(A_{j}^{\prime \prime}, G_{B_{m}}\right)$ exist and do not depend on the reference point $o$. We claim that

$$
\lim _{m \rightarrow \infty}\left(\mathscr{U}^{k} a, G_{B_{m}}\right)=\sum_{i=1}^{d} \lim _{m \rightarrow \infty}\left(A_{i}^{\prime}, G_{B_{m}}\right)+\sum_{j=1}^{\infty} \lim _{m \rightarrow \infty}\left(A_{j}^{\prime \prime}, G_{B_{m}}\right) .
$$

To prove this we set $c_{j, m}:=\left(A_{j}^{\prime \prime}, G_{B_{m}}\right)$ and we show that $\sup _{m}\left|c_{j, m}\right|$ is a summable sequence, whence the result will follow by the Dominated Convergence Theorem. We denote by $\delta$ the distance between $p$ and $o$ and consider the three cases

$$
m<\delta-j-1, \quad \delta-j-1 \leq m \leq \delta+j+1 \quad \text { and } \quad m>\delta+j+1
$$

separately.

In the first case, $B_{j}^{\prime \prime} \cap B_{m}=\emptyset$, so that $c_{j, m}=0$.

In the second case, $B_{j}^{\prime \prime} \cap B_{m} \neq \emptyset$, and

$$
\begin{aligned}
\left|c_{j, m}\right| & \leq\left\|A_{j}^{\prime \prime}\right\|_{2}\left\|G_{B_{m}}\right\|_{2} \\
& \leq C \mathrm{e}^{-2 \beta j} m \mu\left(B_{m}\right)^{1 / 2}\|\mathbf{G}\|_{\mathbb{Y}^{1}} \\
& \leq C \mathrm{e}^{-2 \beta j}(\delta+j+1) \mu\left(B_{\delta+j+1}\right)^{1 / 2}\|\mathbf{G}\|_{\mathbb{Y}^{1}} \\
& \leq C \mathrm{e}^{-\varepsilon j}\|\mathbf{G}\|_{\mathbb{Y}^{1}},
\end{aligned}
$$


for some positive $\varepsilon$. Here we have used the estimate of the $L^{2}$-norm of $A_{j}^{\prime \prime}$ and $G_{B}$ given in Lemma 4.18 (ii) and (ii) above, respectively, and inequality (4.1). The constant $C$ is independent of $j$ and $m$, but may depend on the point $p$.

Finally, in the third case $B_{m} \supset B_{j}^{\prime \prime}$. Since $A_{j}^{\prime \prime}$ is in $q_{1}^{2}\left(B_{j}^{\prime \prime}\right)^{\perp}$ and $\pi_{B_{j}^{\prime \prime}, 1}\left(G_{B_{m}}\right)=$ $G_{B_{j}^{\prime \prime}}$

$$
\begin{aligned}
\left|c_{j, m}\right| & =\left|\left(A_{j}^{\prime \prime}, \pi_{B_{j}^{\prime \prime}, 1}\left(G_{B_{m}}\right)\right)\right| \\
& \leq\left\|A_{j}^{\prime \prime}\right\|_{2}\left\|\pi_{B_{j}^{\prime \prime}, 1}\left(G_{B_{m}}\right)\right\|_{2} \\
& =\left\|A_{j}^{\prime \prime}\right\|_{2}\left\|G_{B_{j}^{\prime \prime}}\right\|_{2} \\
& \leq C \mathrm{e}^{-2 \beta j} j \mu\left(B_{j}^{\prime \prime}\right)^{1 / 2}\|\mathbf{G}\|_{\mathbb{Y}^{1}} \\
& \leq C \mathrm{e}^{-\varepsilon j}\|\mathbf{G}\|_{\mathbb{Y}^{1}},
\end{aligned}
$$

for some positive $\varepsilon$. Here we have applied again the estimate of the $L^{2}$-norms of $A_{j}^{\prime \prime}$ and of $G_{B_{i}^{\prime \prime}}$ given in Lemma 4.18 (ii) and in (ii) above, respectively, and $C$ is a constant which is independent of $j$ and $m$, but may depend on the point $p$.

This completes the proof that $\sup _{m}\left|c_{j, m}\right|$ is a summable sequence. To conclude the proof of point (iii) of the lemma, it remains to prove the estimate in the statement. Since $A_{i}^{\prime}$ and $A_{j}^{\prime \prime}$ are in $q_{1}^{2}\left(B_{i}^{\prime}\right)^{\perp}$ and $q_{1}^{2}\left(B_{j}^{\prime \prime}\right)^{\perp}$, respectively, we get

$$
\begin{aligned}
\left|\lambda\left(\mathscr{U}^{k} a\right)\right| & \leq \sum_{i=1}^{d}\left|\lim _{m \rightarrow \infty}\left(A_{i}^{\prime}, G_{B_{m}}\right)\right|+\sum_{j=1}^{\infty}\left|\lim _{m \rightarrow \infty}\left(A_{j}^{\prime \prime}, G_{B_{m}}\right)\right| \\
& =\sum_{i=1}^{d}\left|\left(A_{i}^{\prime}, G_{B_{i}^{\prime}}\right)\right|+\sum_{j=1}^{\infty}\left|\left(A_{j}^{\prime \prime}, G_{B_{j}^{\prime \prime}}\right)\right| \\
& \leq \sum_{i=1}^{d}\left\|A_{i}^{\prime}\right\|_{2}\left\|G_{B_{i}^{\prime}}\right\|_{2}+C\|\mathbf{G}\|_{\mathbb{Y}^{1}} \sum_{j=1}^{\infty} \mathrm{e}^{-2 \beta j} j \mu\left(B_{j}^{\prime \prime}\right)^{1 / 2} \\
& \leq \sum_{i=1}^{d} 4^{-i} \mu\left(B_{i}^{\prime}\right)^{-1 / 2}\left\|G_{B_{i}^{\prime}}\right\|_{2}+C\|\mathbf{G}\|_{\mathbb{Y}^{1}} \\
& \leq C\|\mathbf{G}\|_{\mathbb{Y}^{1}},
\end{aligned}
$$

where we have applied Lemma 4.18 and $C$ is independent of $a$, as required.

To prove (iv) observe that, given $\mathbf{G}$ is in $\mathbb{Y}^{k}(M)$, the linear functional $a \mapsto$ $\lim _{m \rightarrow \infty}\left(\mathscr{U}^{k} a, G_{B_{m}}\right)$ extends, by (iii), to a unique linear functional on $H_{\text {fin }}^{1}(M)$ that is uniformly bounded on atoms. Thus, it extends to a unique continuous linear functional, $\ell$ say, on $H^{1}(M)$ (see [15, Theorem 4.1]). In particular,

$$
\|\ell\|_{\left(H^{1}\right)^{*}} \leq C \sup \left\{|\ell(a)|: a H^{1} \text {-atom }\right\} \leq C\|\mathbf{G}\|_{\mathbb{Y}^{k}},
$$


where $C$ is the same as in (iii). Since $\mathscr{U}^{-k}$ is an isometry between $X^{k}(M)$ and $H^{1}(M)$, the linear functional $\ell \circ \mathscr{U}^{-k}$ is in $Y^{k}(M)$, and

$$
\left\|\ell \circ \mathscr{U}^{-k}\right\|_{Y^{k}}=\|\ell\|_{\left(H^{1}\right)^{*}} \leq C\|\mathbf{G}\|_{\mathbb{Y}^{k}}
$$

Furthermore, if $F$ is in $\mathscr{U}^{k} H_{\text {fin }}^{1}(M)$, then $F=\mathscr{U}^{k} f$ for some $f$ in $H_{\text {fin }}^{1}(M)$, and

$$
\begin{aligned}
\left(\ell \circ \mathscr{U}^{-k}\right)(F) & =\left(\ell \circ \mathscr{U}^{-k}\right)\left(\mathscr{U}^{k} f\right) \\
& =\ell(f) \\
& =\lim _{m \rightarrow \infty}\left(\mathscr{U}^{k} f, G_{B_{m}}\right),
\end{aligned}
$$

so that $\ell \circ \mathscr{U}^{-k}$ coincides with $\lambda_{\mathbf{G}}$ on $\mathscr{U}^{k} H_{\text {fin }}^{1}(M)$ and

$$
\begin{aligned}
\left|\left(\ell \circ \mathscr{U}^{-k}\right)(F)\right| & =|\ell(f)| \\
& \leq C\|f\|_{H^{1}}\|\mathbf{G}\|_{\mathbb{Y}^{k}} \\
& =C\|F\|_{X^{k}}\|\mathbf{G}\|_{\mathbb{Y}^{k}} \quad \forall F \in \mathscr{U}^{k}\left(H_{\text {fin }}^{1}(M)\right) .
\end{aligned}
$$

Since $X_{\text {fin }}^{k}(M) \subset \mathscr{U}^{k}\left(H_{\text {fin }}^{1}(M)\right)$ (see Remark 4.15), the space $\mathscr{U}^{k}\left(H_{\text {fin }}^{1}(M)\right)$ is dense in $X^{k}(M)$. Then, by (5.6), $\ell \circ \mathscr{U}^{-k}$ extends to a unique bounded functional $\lambda_{\mathbf{G}}$ on $X^{k}(M)$, defined by (5.3) on $X_{\text {fin }}^{k}(M)$, such that $\left\|\lambda_{\mathbf{G}}\right\|_{\left(X^{k}\right)^{*}} \leq C\|\mathbf{G}\|_{\mathbb{Y}^{k}}$.

Finally, we prove (v). Pick $G$ in $\operatorname{GBMO}^{k}(M)$ and set $\mathbf{G}:=\left\{\pi_{B, k}(G): B \in\right.$ $\mathscr{B}$ \}. Clearly, $\iota\left(G+q_{k}(M)\right)$ agrees on $X_{\text {fin }}^{k}(M)$ with the functional $\lambda_{\mathbf{G}}$, defined in (5.3). By (iv), $\lambda_{\mathbf{G}}$ extends uniquely to a continuous linear functional on $X^{k}(M)$ (for $X_{\text {fin }}^{k}(M)$ is norm dense in $X^{k}(M)$ ). The required norm estimate follows then from (iv).

Corollary 5.6. All the spaces $\mathrm{GBMO}_{s}^{k}(M), 0<s<\operatorname{Inj}(M)$, coincide and all the norms $\|\cdot\|_{\mathrm{GBMO}_{s}^{k}}$ are equivalent.

Proof. Suppose that $s_{1} \leq s_{2}$. It is obvious from Definition 5.2 that $\operatorname{GBMO}_{s_{2}}^{k}(M) \subseteq$ $\operatorname{GBMO}_{s_{1}}^{k}(M)$ and $\|G\|_{\mathrm{GBMO}_{s_{1}}^{k}} \leq\|G\|_{\mathrm{GBMO}_{s_{2}}^{k}}$.

Assume next that $G \in \mathrm{GBMO}_{s_{1}}^{k}(M)$ and for every ball $B \in \mathscr{B}$ define $G_{B}=$ $\pi_{B, k}(G)$. Let $\lambda_{G}$ be the linear functional on $X_{\text {fin }}^{k}(M)$ defined by

$$
\lambda_{G}(F)=\lim _{B} \int F G_{B} \mathrm{~d} \mu .
$$

Now, arguing as in the proof of Lemma 5.5 (i), but using $X^{k}$-atoms with support contained in balls of radius less than $s_{1}$ instead of $s_{0}$ (see Remark 4.14), we obtain that

$$
\left|\lambda_{G}(F)\right| \leq C\|G\|_{\mathrm{GBMO}_{s_{1}}^{k}}\|F\|_{X_{\text {fin }}^{k}(M)} \quad \forall F \in X_{\text {fin }}^{k}(M) .
$$


Hence, arguing as in the proof of part (ii) of Lemma 5.5, we obtain that for all balls $B \in \mathscr{B}$

$$
\begin{aligned}
\left\|G_{B}\right\|_{2} & \leq C\left\|\lambda_{G}\right\|_{\left(X_{\text {fin }}^{k}\right)^{*}} C\left(1+r_{B}\right) \mu(B)^{1 / 2} \\
& \leq C\|G\|_{\mathrm{GBMO}_{s_{1}}^{k}}\left(1+r_{B}\right) \mu(B)^{1 / 2} .
\end{aligned}
$$

Thus

$$
\|G\|_{\mathrm{GBMO}_{s_{2}}^{k}}=\sup _{B \in \mathscr{B}_{s_{2}}} \mu(B)^{-1 / 2}\left\|G_{B}\right\|_{2} \leq C\left(1+s_{2}\right)\|G\|_{\mathrm{GBMO}_{s_{1}}^{k}} .
$$

This shows that $\mathrm{GBMO}_{s_{1}}^{k}(M) \subseteq \mathrm{GBMO}_{s_{2}}^{k}(M)$ and that the two norms are equivalent.

To prove that the map $\iota: \operatorname{GBMO}^{k}(M) / q_{k}(M) \rightarrow Y^{k}(M)$ is an isomorphism, we need a regularity result for solutions in $L_{\text {loc }}^{2}(M)$ of the equation $\mathscr{L}^{k} u=g$ with $g$ in $\operatorname{BMO}(M)$.

Proposition 5.7. For every $g \in \mathrm{BMO}(M)$ and for every positive integer $k$ any solution $U_{k, g}$ of the equation $\mathscr{L}^{k} u=g$ is in $\mathrm{GBMO}^{k}(M)$ and

$$
\left\|U_{k, g}\right\|_{\mathrm{GBMO}^{k}} \leq\left\|\mathscr{L}^{-k}\right\|_{2}\|g\|_{\mathrm{BMO}} .
$$

Proof. Suppose that $B$ is in $\mathscr{B}_{s_{0}}$, and denote by $\varphi$ a smooth function with compact support that is equal to 1 in a neighbourhood of $\bar{B}$. Then the function $\mathscr{L}^{-k}(\varphi g)$ satisfies the equation $\mathscr{L}^{k} u=\varphi g$ on $M$. Hence $U_{k, g}-\mathscr{L}^{-k}(\varphi g)$ satisfies the equation $\mathscr{L}^{k} u=(1-\varphi) g$, so that $U_{k, g}-\mathscr{L}^{-k}(\varphi g)$ is $k$-harmonic in a neighbourhood of $\bar{B}$. Therefore

$$
\pi_{B, k}\left(U_{k, g}-\mathscr{L}^{-k}(\varphi g)\right)=0,
$$

whence

$$
\begin{aligned}
\left\|\pi_{B, k}\left(U_{k, g}\right)\right\|_{2} & =\left\|\pi_{B, k}\left(\mathscr{L}^{-k}(\varphi g)\right)\right\|_{2} \\
& =\sup \left\{\left|\left(F, \mathscr{L}^{-k}(\varphi g)\right)\right|: F \in q_{k}^{2}(B)^{\perp},\|F\|_{2}=1\right\} .
\end{aligned}
$$

Since $\mathscr{L}^{-k}$ is self adjoint and $\mathscr{L}^{-k} F$ is a multiple of an $H^{1}$-atom supported in $\bar{B}$ (see [17, Remark 3.5]),

$$
\begin{aligned}
\left(F, \mathscr{L}^{-k}(\varphi g)\right) & =\left(\mathscr{L}^{-k} F, \varphi g\right)=\int_{B} \mathscr{L}^{-k} F g \mathrm{~d} \mu \\
& =\int_{B} \mathscr{L}^{-k} F\left(g-g_{B}\right) \mathrm{d} \mu .
\end{aligned}
$$

This implies that

$$
\left|\left(F, \mathscr{L}^{-k}(\varphi g)\right)\right| \leq\left|\int_{B} \mathscr{L}^{-k} F\left(g-g_{B}\right) \mathrm{d} \mu\right| \leq\left\|\mathscr{L}^{-k}\right\|_{2}\left\|g-g_{B}\right\|_{L^{2}(B)} .
$$


Thus

$$
\sup \left\{\left|\left(F, \mathscr{L}^{-k}(\varphi g)\right)\right|: F \in q_{k}^{2}(B)^{\perp},\|F\|_{2}=1\right\} \leq\left\|\mathscr{L}^{-k}\right\|_{2}\left\|g-g_{B}\right\|_{L^{2}(B)} .
$$

By combining this inequality with the formulae above, we conclude that

$$
\begin{aligned}
\left\|U_{k, g}\right\|_{\mathrm{GBMO}^{k}} & =\sup _{B \in \mathscr{B}_{s_{0}}} \mu(B)^{-1 / 2}\left\|\pi_{B, k}\left(U_{k, g}\right)\right\|_{2} \\
& \leq\left\|\mathscr{L}^{-k}\right\|_{2} \sup _{B \in \mathscr{B}_{s_{0}}} \mu(B)^{-1 / 2}\left\|g-g_{B}\right\|_{L^{2}(B)} \\
& =\left\|\mathscr{L}^{-k}\right\|_{2}\|g\|_{\mathrm{BMO}},
\end{aligned}
$$

as required.

Theorem 5.8. Suppose that $k$ is a positive integer and that $M$ has $C^{2 k-2}$ bounded geometry. Then the map ı (see Lemma $5.5(\mathrm{v})$ ) is a Banach space isomorphism between $\mathrm{GBMO}^{k}(M) / q_{k}(M)$ and $Y^{k}(M)$.

Proof. By Lemma 5.5 (v) the map $\iota$ is continuous. We shall prove that $\iota$ is bijective. The required conclusion will then follow from (a standard consequence of) the Open Mapping Theorem.

First we show that $\iota$ is injective. Suppose that $G$ is a function in $\operatorname{GBMO}^{k}(M)$ such that $\iota\left(G+q_{k}(M)\right)=0$. In particular,

$$
\iota\left(G+q_{k}(M)\right)(A)=\int_{M} A G \mathrm{~d} \mu=0
$$

for every $X^{k}$-atom $A$. This implies that $\pi_{B, k}(G)=0$ for every ball $B$ with radius $\leq s_{0}$. By Proposition 5.1, $G$ is in $q_{k}(M)$, as required.

Next we prove that $\iota$ is surjective. Suppose that $\lambda$ is in $Y^{k}(M)$. Since $\left(\mathscr{U}^{-k}\right)^{t}$ is an isomorphism between $\operatorname{BMO}(M) / \mathbb{C}$ and $Y^{k}(M)$, there exists a unique coset $g+\mathbb{C}$ in $\operatorname{BMO}(M) / \mathbb{C}$ such that

$$
\left(\mathscr{U}^{-k}\right)^{t}(g+\mathbb{C})=\lambda .
$$

Therefore, for every $X^{k}$-atom $A$

$$
\lambda(A)=\left\langle A,\left(\mathscr{U}^{-k}\right)^{t}(g+\mathbb{C})\right\rangle=\left\langle\mathscr{U}^{-k}(A), g+\mathbb{C}\right\rangle,
$$

by definition of transpose operator. The pairing in the first line is the duality between $X^{k}(M)$ and $Y^{k}(M)$ and that in the second is the duality between $H^{1}(M)$ and $\operatorname{BMO}(M) / \mathbb{C}$. Since $A$ is in $L^{2}(M)$,

$$
\mathscr{U}^{-k}(A)=\sum_{j=0}^{k}\left(\begin{array}{l}
k \\
j
\end{array}\right) \sigma^{j} \mathscr{L}^{-j} A .
$$


Since $A$ is an $X^{k}$-atom, then $A$ is an $X^{j}$-atom for every $j=0, \ldots, k$. By [17, Remark 3.5] $\mathscr{L}^{-j} A$ is a multiple of an $H^{1}$-atom, so in particular $\mathscr{L}^{-j} A, j=$ $0,1, \ldots, k$, are in $H_{\text {fin }}^{1}(M)$. Hence

$$
\left\langle\mathscr{U}^{-k}(A), g+\mathbb{C}\right\rangle=\int_{M}\left(\sum_{j=0}^{k}\left(\begin{array}{l}
k \\
j
\end{array}\right) \sigma^{j} \mathscr{L}^{-j} A\right) g \mathrm{~d} \mu .
$$

Now, denote by $\varphi$ a smooth function with compact support which is equal to 1 in a neighbourhood of the support of $A$, and let $U_{g, j}$ denote any global solution of the equation $\mathscr{L}^{j} u=g$. We remark that $U_{g, j} \in \operatorname{GBMO}^{j}(M) \subseteq \mathrm{GBMO}^{k}(M)$ by Proposition 5.7 and the remark preceeding (5.1). Then

$$
\begin{aligned}
\int_{M} \mathscr{L}^{-j} A g \mathrm{~d} \mu & =\int_{M} \mathscr{L}^{-j} A \mathscr{L}^{j} U_{g, j} \mathrm{~d} \mu=\int_{M} \mathscr{L}^{-j} A \mathscr{L}^{j}\left(\varphi U_{g, j}\right) \mathrm{d} \mu \\
& =\int_{M} A \varphi U_{g, j} \mathrm{~d} \mu=\int_{M} A U_{g, j} \mathrm{~d} \mu .
\end{aligned}
$$

We have used the fact that the support of $\mathscr{L}^{-j} A$ is contained in the support of $A$ in the second equality, and the self adjointness of $\mathscr{L}$ in the third equality. Now, define $U=\sum_{j=0}^{k}\left(\begin{array}{c}k \\ j\end{array}\right) \sigma^{j} U_{g, j}$. The function $U$ is in $\operatorname{GBMO}^{k}(M)$ because $U_{g, j} \in$ $\mathrm{GBMO}^{j}(M) \subseteq \mathrm{GBMO}^{k}(M)$ for all $j=0, \ldots, k$. By combining the formulae above, we see that

$$
\lambda(A)=\int_{M} A U \mathrm{~d} \mu=\iota\left(U+q_{k}(M)\right)(A) .
$$

This completes the proof of the surjectivity of $\iota$, and of the theorem.

Remark 5.9. We observe that in the proof of Theorem 5.9 we have actually shown the commutativity of the following diagram

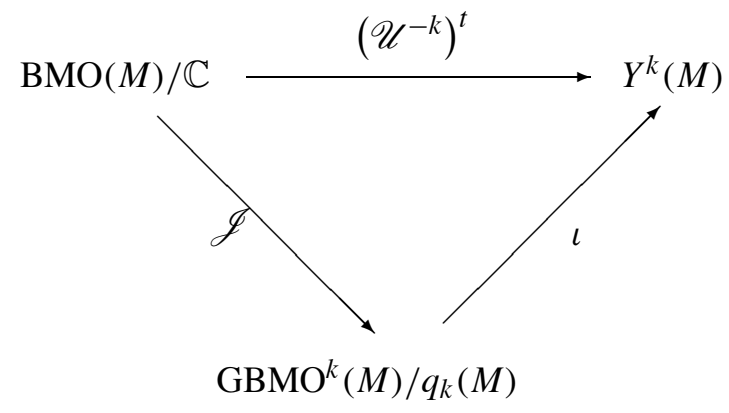

where $\mathscr{J}$ is the map $g+\mathbb{C} \mapsto \sum_{j=0}^{k}\left(\begin{array}{l}k \\ j\end{array}\right) \sigma^{j} U_{g, j}+q_{k}(M)$.

Now we draw a few consequences of Theorem 5.8. 
Corollary 5.10. If $g$ is a function in $\mathrm{BMO}(M)$ such that $\mathscr{L} g+\sigma g=$ const then $g$ is constant.

Proof. Let $U_{g, 1}$ be a global solution of the equation $\mathscr{L} u=g$. Then $g+\sigma U_{g, 1} \in$ $q_{1}(M)$. Thus

$$
\int_{M}\left(g+\sigma U_{g, 1}\right) A \mathrm{~d} \mu=0
$$

for all $X^{1}$-atoms $A$. In the proof of Theorem 5.8 we have shown that

$$
\left\langle A,\left(\mathscr{U}^{-1}\right)^{t}(g+\mathbb{C})\right\rangle=\left\langle\mathscr{U}^{-1} A, g+\mathbb{C}\right\rangle=\int_{M}\left(g+\sigma U_{g, 1}\right) A \mathrm{~d} \mu=0 .
$$

Thus $\left(\mathscr{U}^{-1}\right)^{t}(g+\mathbb{C})=0$ and the conclusion follows, since $\left(\mathscr{U}^{-1}\right)^{t}$ is an isomorphism.

Corollary 5.11. For every $\mathbf{G}=\left\{G_{B}: B \in \mathscr{B}\right\}$ in $\mathbb{Y}^{k}(M)$, there exists $G$ in $\mathrm{GBMO}^{k}(M)$ such that $G_{B}=\pi_{B, k}(G)$ for every $B$ in $\mathscr{B}$. Furthermore, $\|\mathbf{G}\|_{\mathbb{Y}^{k}} \asymp$ $\|G\|_{\mathrm{GBMO}^{k}}$.

Proof. Suppose that $\mathbf{G}$ is in $\mathbb{Y}^{k}(M)$. By Lemma 5.5 (iv), the linear functional $\lambda_{\mathbf{G}}$, defined by

$$
\lambda_{\mathbf{G}}(F)=\lim _{B^{\prime}} \int_{M} F G_{B^{\prime}} \mathrm{d} \mu \quad \forall F \in X_{\text {fin }}^{k}(M),
$$

is in the dual $Y^{k}(M)$ of $X^{k}(M)$. Theorem 5.8 then ensures the existence of a function $G$ in $\operatorname{GBMO}^{k}(M)$ such that $\lambda_{\mathbf{G}}=\iota\left(G+q_{k}(M)\right)$, and

$$
\iota\left(G+q_{k}(M)\right)(F)=\int_{M} F G \mathrm{~d} \mu \quad \forall F \in X_{\text {fin }}^{k}(M) .
$$

Therefore, given a ball $B$, for every ball $B^{\prime}$ containing $B$ and for every (possibly not admissible) $X^{k}$-atom $A$ associated to $B$ we have that

$$
\int_{M} A G_{B^{\prime}} \mathrm{d} \mu=\int_{M} A G \mathrm{~d} \mu .
$$

It follows that $\pi_{B, k}\left(G_{B^{\prime}}\right)=\pi_{B, k}(G)$. But $\pi_{B, k}\left(G_{B^{\prime}}\right)=G_{B}$, because $\mathbf{G}$ is in $\mathbb{Y}^{k}(M)$, and the required formula follows. The equivalence of the norms of $\mathbf{G}$ and $G$ is an obvious consequence of the definition of the "norms" of $\mathbb{Y}^{k}(M)$ and $\mathrm{GBMO}^{k}(M)$.

\section{The dual of $X_{\text {fin }}^{k}(M)$}

A noteworthy consequence of the theory developed in Section 5 is the fact, proved in the next theorem, that $X_{\text {fin }}^{k}(M)$ and $X^{k}(M)$ have isomorphic duals. 
Theorem 6.1. Suppose that $k$ is a positive integer and $M$ has $C^{2 k-2}$ bounded geometry. The dual of $X_{\text {fin }}^{k}(M)$ is isomorphic to $\operatorname{GBMO}^{k}(M) / q_{k}(M)$. The continuous linear functionals on $X_{\text {fin }}^{k}(M)$ are precisely those of the form

$$
\lambda_{G}(F)=\int_{M} F G \mathrm{~d} \mu \quad \forall F \in X_{\text {fin }}^{k}(M)
$$

for $G$ in $\mathrm{GBMO}^{k}(M)$. Furthermore $\|\lambda\|_{\left(X_{\text {fin }}^{k}\right)^{*}} \asymp\|G\|_{\mathrm{GBMO}^{k}}$.

Proof. By Lemma 5.5 (i) the linear functional $\lambda_{G}$ is continuous on $X_{\text {fin }}^{k}(M)$ and $\left\|\lambda_{G}\right\|_{\left(X_{\text {fin }}^{k}\right)^{*}} \leq\|G\|_{\mathrm{GBMO}^{k}}$.

Conversely, given a continuous linear functional $\lambda$ on $X_{\text {fin }}^{k}(M)$, for every $B$ in $\mathscr{B}$ the restriction of $\lambda$ to $q_{k}^{2}(B)^{\perp}$ is in $\left(q_{k}^{2}(B)^{\perp}\right)^{*}$. Indeed,

$$
\begin{aligned}
|\lambda(F)| & \leq\|\lambda\|_{\left(X_{\text {fin }}^{k}\right)^{*}}\|F\|_{X_{\text {fin }}^{k}} \\
& \leq C\|\lambda\|_{\left(X_{\text {fin }}^{k}\right)^{*}}\left(1+r_{B}\right) \mu(B)^{1 / 2}\|F\|_{2} \quad \forall F \in q_{k}^{2}(B)^{\perp},
\end{aligned}
$$

where we have used Lemma 4.17. By the Riesz Representation Theorem, there exists $G_{B}$ in $q_{k}^{2}(B)^{\perp}$ such that

$$
\lambda(F)=\left(F, G_{B}\right) \quad \forall F \in q_{k}^{2}(B)^{\perp},
$$

where $(\cdot, \cdot)$ denotes the inner product in $q_{k}^{2}(B)^{\perp}$, i.e., the restriction to $q_{k}^{2}(B)^{\perp}$ of the inner product in $L^{2}(B)$. Furthermore $\left\|\lambda_{\mid q_{k}^{2}(B)^{\perp}}\right\|=\left\|G_{B}\right\|_{2}$. By combining this and (6.1), we obtain that

$$
\left\|G_{B}\right\|_{2} \leq C\|\lambda\|_{\left(X_{\text {fin }}^{k}\right)^{*}}\left(1+r_{B}\right) \mu(B)^{1 / 2},
$$

where $C$ is independent of $B$. Taking the supremum over all balls $B$ in $\mathscr{B}_{s_{0}}$ we obtain

$$
\sup _{B \in \mathscr{B}_{s_{0}}}\left(\frac{1}{\mu(B)} \int_{B}\left|G_{B}\right|^{2} \mathrm{~d} \mu\right)^{1 / 2} \leq C\|\lambda\|_{\left(X_{\text {fin }}^{k}\right)^{*}} .
$$

Suppose that $B, B^{\prime}$ are balls such that $B \subset B^{\prime}$ and identify $L^{2}(B)$ with the subspace of all functions in $L^{2}\left(B^{\prime}\right)$ that vanish on $B^{\prime} \backslash B$. Then $q_{k}^{2}(B)^{\perp} \subset q_{k}^{2}\left(B^{\prime}\right)^{\perp}$ and

$$
\int_{B} F G_{B} \mathrm{~d} \mu=\int_{B^{\prime}} F G_{B^{\prime}} \mathrm{d} \mu \quad \forall F \in q_{k}^{2}(B)^{\perp} .
$$

Hence $G_{B}=\pi_{B, k}\left(G_{B^{\prime}}\right)$. As a consequence, $\mathbf{G}:=\left\{G_{B}: B \in \mathscr{B}\right\}$ is in $\mathbb{Y}^{k}(M)$, and

$$
\|\mathbf{G}\|_{\mathbb{Y}^{k}} \leq C\|\lambda\|_{\left(X_{\text {fin }}^{k}\right)^{*}}
$$

By Corollary 5.11 there exists $G$ in $\operatorname{GBMO}^{k}(M)$ such that $G_{B}=\pi_{k, B}(G)$ and $\|\mathbf{G}\|_{\mathbb{Y}^{k}}=\|G\|_{\mathrm{GBMO}^{k}}$. Therefore $\lambda$ agrees with $\lambda_{G}$. 
A corollary of the theory we developed is the following.

Corollary 6.2. If $k$ is a positive integer and $M$ has $C^{2 k-2}$ bounded geometry then the following hold:

(i) the $X_{\text {fin }}^{k}$-norm and the $X^{k}$-norm are equivalent on $X_{\text {fin }}^{k}(M)$;

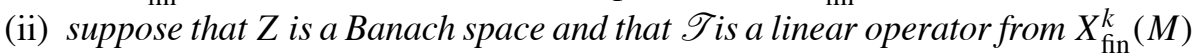
into $Z$, such that

$$
L:=\sup \left\{\|\mathscr{T} A\|_{Z}: A \text { admissible } X^{k} \text {-atom }\right\}<\infty .
$$

Then $\mathscr{T}$ extends to a unique bounded linear operator from $X^{k}(M)$ to $Z$.

Proof. Part (i) follows directly from the fact that $X_{\text {fin }}^{k}(M)$ and $X^{k}(M)$ have isomorphic dual spaces.

To prove (ii) observe that a direct consequence of the assumption is that if $F=\sum_{j=1}^{N} c_{j} A_{j}$ is a function in $X_{\text {fin }}^{k}(M)$, then, by the triangle inequality,

$$
\|\mathscr{T} F\|_{Z} \leq L \sum_{j=1}^{N}\left|c_{j}\right| .
$$

By taking the infimum over all representations of $F$ as a finite linear combination of $X^{k}$-atoms, we obtain

$$
\|\mathscr{T} F\|_{Z} \leq L\|F\|_{X_{\text {fin }}^{k}} \leq C\|F\|_{X^{k}} \quad \forall F \in X_{\text {fin }}^{k}(M) .
$$

We have used (i) in the second inequality above. The required conclusion follows from the density of $X_{\text {fin }}^{k}(M)$ in $X^{k}(M)$.

Quite often one encounters the following situation. Suppose that $\mathscr{T}$ is a bounded linear operator on $L^{2}(M)$. Then $\mathscr{T}$ is automatically defined on $X_{\text {fin }}^{k}(M)$. Assume that

$$
L:=\sup \left\{\|\mathscr{T} A\|_{L^{1}}: A \text { admissible } X^{k}-\text { atom }\right\}<\infty .
$$

By the previous results, the restriction of $\mathscr{T}$ to $X_{\text {fin }}^{k}(M)$ has a unique extension to a bounded linear operator $\widetilde{\mathscr{T}}$ from $X^{k}(M)$ to $L^{1}(M)$. The question is whether the operators $\mathscr{T}$ and $\widetilde{\mathscr{T}}$ are consistent, i.e., whether they coincide on the intersection $X^{k}(M) \cap L^{2}(M)$ of their domains.

Proposition 6.3. Suppose that $k$ is a positive integer and $M$ has $C^{2 k-2}$ bounded geometry and that $\mathscr{T}$ is bounded on $L^{2}(M)$. The following hold:

(i) if $L_{0}:=\sup \left\{\|\mathscr{T a}\|_{L^{1}}: a\right.$ admissible $H^{1}-$ atom $\}<\infty$, then the unique continuous linear extension $\widetilde{\mathscr{T}}$ of the restriction of $\mathscr{T}$ to $H_{\text {fin }}^{1}(M)$ to an operator from $H^{1}(M)$ to $L^{1}(M)$ agrees with $\mathscr{T}$ on $H^{1}(M) \cap L^{2}(M)$; 
(ii) if $L:=\sup \left\{\|\mathscr{T} A\|_{L^{1}}: A\right.$ admissible $X^{k}-$ atom $\}<\infty$, then

$$
\sup \left\{\left\|\mathscr{T} \mathscr{U}^{k} a\right\|_{L^{1}}: a \text { admissible } H^{1}-\text { atom }\right\}<\infty ;
$$

(iii) if $L:=\sup \left\{\|\mathscr{T} A\|_{L^{1}}: A\right.$ admissible $X^{k}$-atom $\}<\infty$, then the unique continuous linear extension $\widetilde{\mathscr{T}}$ of the restriction of $\mathscr{T}$ to $X_{\text {fin }}^{k}(M)$ to an operator from $X^{k}(M)$ to $L^{1}(M)$ agrees with $\mathscr{T}$ on $X^{k}(M) \cap L^{2}(M)$.

Proof. The proof of (i) follows the same line of the proof of [20, Proposition 4.2], and is omitted.

We give the proof of (ii) for $k=1$. The proof in the case where $k \geq 2$ is similar and is omitted.

Suppose that the atom $a$ is supported in the ball $B(p, R)$ with $R \leq s_{0}$. The proof hinges on the decomposition

$$
\mathscr{U} a=\sum_{i=0}^{d} A_{i}^{\prime}+\sum_{j=1}^{\infty} A_{j}^{\prime \prime},
$$

given in Lemma 4.18. The function $4^{i} A_{i}^{\prime}$ is a multiple of an admissible $X^{1}$-atom. Then

$$
\left\|\mathscr{T}\left(4^{i} A_{i}^{\prime}\right)\right\|_{L^{1}} \leq C L .
$$

Thus,

$$
\left\|\sum_{i=0}^{d} \mathscr{T} A_{i}^{\prime}\right\|_{L^{1}} \leq C L \sum_{i=0}^{d} 4^{-i} \leq C L,
$$

where $C$ is independent of $a$.

For every $j$ in $\mathbb{N}$ by (4.4) we have

$$
\left\|\mathscr{T} A_{j}^{\prime \prime}\right\|_{L^{1}} \leq C \mathrm{e}^{-\varepsilon j} L
$$

Thus,

$$
\sum_{j=1}^{\infty}\left\|\mathscr{T} A_{j}^{\prime \prime}\right\|_{L^{1}} \leq C L \sum_{j=1}^{\infty} \mathrm{e}^{-\varepsilon j} \leq C L .
$$

The inequalities (6.3) and (6.4) imply that

$$
\|\mathscr{T} \mathscr{U} a\|_{L^{1}} \leq C L,
$$

as required. 
Finally, we prove (iii). We consider the operator $\mathscr{T} \circ \mathscr{U}^{k}$, which is bounded on $L^{2}(M)$ and uniformly bounded on admissible $H^{1}$-atoms by (ii). By (i) the unique extension $\widetilde{\mathscr{T} \circ \mathscr{U}^{k}}$ of the restriction of $\mathscr{T} \circ \mathscr{U}^{k}$ to $H_{\text {fin }}^{1}(M)$ to an operator bounded from $H^{1}(M)$ to $L^{1}(M)$ agrees with $\mathscr{T} \circ \mathscr{U}^{k}$ on $H^{1}(M) \cap L^{2}(M)$.

Then the operator $\left(\widetilde{\mathscr{T} \circ \mathscr{U}^{k}}\right) \circ \mathscr{U}^{-k}$ is a bounded operator from $X^{k}(M)$ to $L^{1}(M)$ which extends the restriction of $\mathscr{T}$ to $X_{\text {fin }}^{k}(M)$. Then it coincides with the unique continuous linear extension $\widetilde{\mathscr{T}}$ of the restriction of $\mathscr{T}$ to $X_{\text {fin }}^{k}(M)$, i.e., $\widetilde{\mathscr{T}}=\left(\widetilde{\mathscr{T} \circ \mathscr{U}^{k}}\right) \circ \mathscr{U}^{-k}$.

Moreover, for every function $F$ in $X^{k}(M) \cap L^{2}(M)$ we have that $\mathscr{U}^{-k} F$ is in $H^{1}(M) \cap L^{2}(M)$. Then

$$
\widetilde{\mathscr{T}} F=\left(\widetilde{\mathscr{T} \circ \mathscr{U}^{k}}\right) \circ \mathscr{U}^{-k} F=\left(\mathscr{T} \circ \mathscr{U}^{k}\right) \circ \mathscr{U}^{-k} F=\mathscr{T} F .
$$

Hence $\widetilde{\mathscr{T}}$ agrees with $\mathscr{T}$ on $X^{k}(M) \cap L^{2}(M)$.

\section{References}

[1] R. AdAms and J. F. J. Fournier, "Sobolev Spaces", Second edition, Pure and Applied Mathematics, Vol. 140, Elsevier/ Academic Press, Amsterdam, 2003.

[2] P. Auscher, A. McIntosh and E. Russ, Hardy spaces of differential forms on Riemannian manifolds, J. Geom. Anal. 18 (2008), 192-248.

[3] T. BAGBY and P. BLANCHET, Uniform harmonic approximation on Riemannian manifolds, J. Anal. Math. 62 (1994), 257-297.

[4] R. BROOKS, A relation between growth and the spectrum of the Laplacian, Math. Z. 178 (1981), 501-508.

[5] A. CARbonaro, G. MAUCERI and S. MedA, $H^{1}$ and BMO for certain locally doubling metric measure spaces, Ann. Sc. Norm. Super. Pisa Cl. Sci. 8 (2009), 543-582.

[6] A. Carbonaro, G. Mauceri and S. Meda, $H^{1}$ and BMO for certain locally doubling metric measure spaces of finite measure, Colloq. Math. 118 (2010), 13-41.

[7] A. Carbonaro, A. MCIntosh and A. J. Morris, Local Hardy Spaces of Differential Forms on Riemannian Manifolds, J. Geom. Anal. 23 (2013), 106-169.

[8] R. R. COIFMAN and G. WEISS, Extensions of Hardy spaces and their use in analysis, Bull. Amer. Math. Soc. 83 (1977), 569-645.

[9] L. C. Evans, "Partial Differential Equations", Graduate Studies in Mathematics, Vol. 19, American Mathematical Society, Providence, RI, 1998.

[10] C. FefFERMAN and E. M. STEIn, $H^{p}$ spaces of several variables, Acta Math. 179 (1972), 137-193.

[11] D. GoldBerg, A local version of real Hardy spaces, Duke Math. J. 46 (1979), 2742.

[12] E. Hebey, "Sobolev Spaces on Riemannian Manifolds", Lecture Notes in Mathematics, Vol. 1635, Springer Verlag, Berlin, 1996.

[13] J.-L. Lions and E. Magenes, Problemi ai limiti non omogenei, Ann. Scuola Norm Sup. Pisa Cl. Sci. (3) 16 (1962), 1-44.

[14] M. MARIAS and E. RUSS, $H^{1}$ boundedness of Riesz transforms and imaginary powers of the Laplacian on Riemanian manifolds, Ark. Mat. 41 (2003), 115-132. 
[15] G. MAUCERI and S. MEDA, Equivalence of norms on finite linear combinations of atoms, Math. Z. 269 (2011), 253-260.

[16] G. MAUCERI, S. MEDA and M. VAllarino, Hardy-type spaces on certain noncompact manifolds and applications, J. Lond. Math. Soc. (2) 84 (2011), 243-268.

[17] G. Mauceri, S. Meda and M. Vallarino, Atomic decomposition of Hardy type spaces on certain noncompact manifolds, J. Geom. Anal. 22 (2012), 864-891.

[18] G. MAUCERI, S. MEDA and M. VALlARINO, Sharp endpoint results for imaginary powers and Riesz transforms on certain noncompact manifolds, Studia Math. 224 (2014), 153-168.

[19] G. MAUCERI, S. Meda and M. VAllarino, Endpoint results for Riesz transforms and spherical multipliers on noncompact symmetric spaces, arXiv:1507.04855.

[20] S. MEDA, P. SJÖGREN and M. VALLARINO, On the $H^{1}-L^{1}$ boundedness of operators, Proc. Amer. Math. Soc. 136 (2008), 2921-2931.

[21] S. Meda, P. SJÖGREN and M. VAllarino, Atomic decompositions and operators on Hardy spaces, Rev. Un. Mat. Argentina 60 (2009), 15-22.

[22] E. RUSS, $H^{1}-L^{1}$ boundedness of Riesz transforms on Riemannian manifolds and on graphs, Potential Anal. 14 (2001), 301-330.

[23] E. M. STEIN, "Topics in Harmonic Analysis Related to the Littlewood-Paley Theory", Annals of Math. Studies, Vol. 63, Princeton, NJ, 1970.

[24] E. M. STEIN, "Harmonic Analysis. Real Variable Methods, Orthogonality and Oscillatory Integrals", Princeton Math. Series Vol. 43, Princeton, NJ, 1993.

[25] R. S. STRICHARTZ, Analysis of the Laplacian on a complete Riemannian manifold, J. Funct. Anal. 52 (1983), 48-79.

[26] M. E. TAYLOR, Hardy spaces and BMO on manifolds with bounded geometry, J. Geom. Anal. 19 (2009), 137-190.

[27] S. VolPI, "Bochner-Riesz Means of Eigenfunction Expansions and Local Hardy Spaces on Manifolds with Bounded Geometry", Ph.D Thesis, Università di MilanoBicocca, 2011.

\author{
Dipartimento di Matematica \\ Università di Genova \\ Via Dodecaneso 35 \\ 16146 Genova, Italia \\ mauceri@dima.unige.it \\ Dipartimento di Matematica \\ e Applicazioni \\ Università di Milano-Bicocca \\ Via R. Cozzi 53 \\ I-20125 Milano, Italia \\ stefano.meda@unimib.it \\ Dipartimento di Scienze Matematiche \\ Politecnico di Torino \\ Corso Duca degli Abruzzi, 24 \\ I-10129 Torino, Italia \\ maria.vallarino@polito.it
}

